TRANSACTIONS OF THE

AMERICAN MATHEMATICAL SOCIETY

Volume 355, Number 5, Pages 1869-1903

S 0002-9947(03)03224-0

Article electronically published on January 10, 2003

\title{
STEENROD OPERATIONS IN CHOW THEORY
}

\author{
PATRICK BROSNAN
}

\begin{abstract}
An action of the Steenrod algebra is constructed on the mod $p$ Chow theory of varieties over a field of characteristic different from $p$, answering a question posed in Fulton's Intersection Theory. The action agrees with the action of the Steenrod algebra used by Voevodsky in his proof of the Milnor conjecture. However, the construction uses only basic functorial properties of equivariant intersection theory.
\end{abstract}

\section{INTRODUCTION}

Let $X$ be a complete complex algebraic variety, let $H_{*}^{B M}(X)$ denote its BorelMoore homology with coefficients in the field $\mathbf{F}_{p}$, and let $S_{\bullet}: H_{*}^{B M}(X) \rightarrow H_{*}^{B M}(X)$ be the total Steenrod $p$-th power operation in Borel-Moore homology. In [4] (Example 19.1.8), Fulton gives a short proof of a theorem of Kawai [6], which says that $S \bullet$ preserves algebraic classes.

Fulton's argument is as follows: Consider a subvariety $V$ of $X$. By Hironaka, there is a resolution of singularities $\pi: M \rightarrow V$. If $\mu_{M}$ is the orientation class of $M$, then the cycle class $\operatorname{cl}(V)$ is equal to $\pi_{*} \mu_{M}$. Let $\psi: H^{*} M \rightarrow H^{*}(T M, T M-0)$ be the Thom isomorphism, and let $S^{\bullet}$ be the total Steenrod operation in singular cohomology. Let $w\left(T_{M}\right)=\psi^{-1} S^{\bullet} \psi(1)$. Fulton gives a formula

$$
S_{\bullet}(\operatorname{cl}(V))=\pi_{*} S_{\bullet}\left(\mu_{M}\right)=\pi_{*}\left(w\left(T_{M}\right)^{-1} \cap \mu_{M}\right) .
$$

Since $w$ can be expressed in terms of the Chern classes of $T M$ [7], it is tempting to use (11) as a definition of $S_{\bullet}$ in the $\bmod p$ Chow groups $A_{*} X \otimes \mathbf{F}_{p}$. The problem, as Fulton notes, is whether $\pi_{*}\left(w\left(T_{M}\right)^{-1} \cap \mu_{M}\right)$ is independent of the resolution $M$.

In this paper, $S_{\bullet}$ is defined for quasi-projective varieties over a field $k$ of characteristic not equal to $p$ using the equivariant extension of Fulton-MacPherson intersection theory developed by Edidin and Graham 3]. (The definition actually works for any algebraic space over $k$ with an injective morphism into a smooth algebraic space, and we extend it to all varieties over $k$ by using a Chow envelope argument.) The construction loosely follows the construction of cohomology operations given by Steenrod and Epstein [8]. The definition is then shown to agree with (11), proving that $\pi_{*}\left(w\left(T_{M}\right)^{-1} \cap \mu_{M}\right)$ is indeed independent of $M$. The paper ends with a demonstration of the Adem relations in the "algebraic Steenrod algebra" generated by the graded components of $S_{\bullet}$. One aspect of our method that may be interesting is that, while Fulton's question definitely involves resolutions of singularities, our construction of $S_{\bullet}$ does not.

Received by the editors January 10, 2000 and, in revised form, September 15, 2000.

2000 Mathematics Subject Classification. Primary 14C25; Secondary 55N91. 
It should be noted that Voevodsky has defined similar operations in the context of motivic cohomology [11], [10. To the extent that motivic cohomology is an extension of the Chow groups, Voevodsky's operations are more general than the ones defined here. However, the construction in this paper fits into the FultonMacPherson framework for intersection theory, and, thus, avoids the technicalities inherent in any discussion of motivic cohomology.

1.1. Outline. I now describe the organization of this paper. Sections 2 and 3 collect some generalities concerning equivariant intersection theory. Unfortunately, to discuss some of the functorial properties of equivariant intersection theory that are needed in the construction of Steenrod operations, it is convenient (if not necessary) to reformulate the definition of the equivariant Chow groups given in [3]. Given this more transparently functorial definition, the properties we need are straightforward but tedious to prove. The reader willing to take these properties for granted may want to skip or skim these two sections.

In section 4 , the notion of the equivariant cycle class of an equivariant cycle is defined. In section 5 this notion is applied to the class $Z^{\times p} \in A_{p k}^{S(p)} X^{p}$ where $Z$ is a dimension $k$ cycle in a variety $X$ and $A_{p k}^{S(p)} X^{p}$ represents the dimension $p k$ equivariant Chow group of $X^{p}$ with the symmetric group $S(p)$ acting on $X^{p}$ by permutations. This equivariant cycle class and its restriction to the equivariant Chow group $A_{p k}^{C(p)} X^{p}$ (where $C(p)$ is the cyclic group of order $p$ ) determine the action of the Steenrod operations on the class of $Z$.

If $X$ were smooth, we would proceed by pulling back the class $Z^{\times p} \in A_{p k}^{C(p)} X^{p}$ to $A_{*}^{C(p)} X$ (where here $C(p)$ acts trivially on $X$ ) via the diagonal map $\Delta: X \rightarrow X^{p}$. This is essentially the step that was taken by Steenrod and Epstein 8 . However, when $X$ is not smooth, we cannot generally pull back via the diagonal map on $X$. Fulton-MacPherson intersection theory allows us to remedy the problem using the following trick: Embed $X$ in a smooth space $W$ and form the diagram

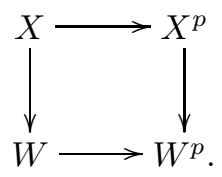

We can then pull back from $A_{p k}^{C(p)} X^{p}$ to $A_{*}^{C(p)} X$ via the refined Gysin map $\Delta_{W}^{!}$. In section 6 this construction is described in detail.

To analyze the resulting pull-back class $\Delta_{W}^{!} Z^{\times p} \in A_{*}^{C(p)} X$, we need to understand the equivariant Chow group $A_{*}^{C(p)} X$. Fortunately, these are very simple. For $k=\mathbf{C}, A_{*}^{C(p)} X=A_{*} X \otimes \mathbf{F}_{p}[l]$ with $l \in A_{-1} X$. In section 7 we state this result (which is given in 9]) and make the appropriate modification for arbitrary fields $k$.

In section 8 , we decompose $\Delta_{W}^{!} Z^{\times p}$ as a polynomial in $\mathbf{F}_{p}[l]$ with coefficients in $A_{*} X$. Re-indexing the coefficients, we arrive at a total Steenrod power $S_{\bullet}^{W}[Z]$. The one defect of this power operation is that it depends on the smooth space $W$. Fortunately, this dependence can be explicitly described in terms of Chern classes of $W$. Thus, we can factor it out and obtain the class $S_{\bullet}[Z]$ - the total Steenrod operation in Chow theory.

In section 9 , we study $S_{\bullet}$ and show that it agrees with the topological $S_{\bullet}$ on Borel-Moore homology. Section 10 extends the definition of $S_{\bullet}$ to varieties $X$ that 
do not admit an embedding $X \rightarrow W$ with $W$ smooth. The Chow envelope argument that allows us to do this is almost identical to an argument used by Fulton in [4] in connection with the Grothendiek-Riemann-Roch theorem. Finally, section 11 proves the Adem relations.

1.2. Conventions. If $\Lambda$ is a commutative ring, the Chow group (resp. the Chow ring) with coefficients in $\Lambda$ is simply $A_{*} X \otimes \Lambda$ (resp. $A^{*} X \otimes \Lambda$ ). Until section 8 all work will be over an arbitrary ring $\Lambda$. Mention of $\Lambda$ will however be suppressed from the notation, with $A_{*} X$ written instead of $A_{*} X \otimes \Lambda$. In section 8 , a prime $p$ will be chosen, and $\Lambda$ will then be set equal to $\mathbf{F}_{p}$.

All schemes (or algebraic spaces) will be assumed to be over a field $k$ whose spectrum will be written pt. We will use the notation $|X|$ to denote the maximum of the dimensions of the connected components of an algebraic space $X$.

\section{Equivariant Intersection Theory I}

The goal of this section is to review the equivariant intersection theory of TotaroEdidin-Graham [9], 3] and to rephrase it slightly in a form more convenient for studying issues of functoriality which arise in the construction of Steenrod powers. Recall that the Borel construction defines the equivariant cohomology of a topological space $X$ with $G$-action as $H^{*}(E G \times X / G)$ where $E G$ is a null-homotopic space on which $G$ acts freely and $E G \times X / G$ is the quotient of the diagonal $G$-action on $E G \times X$.

The idea of Totaro 9 is to replace the topologist's $E G$ by a suitable algebraic analogue. Let $G$ now denote a linear algebraic group over a field $k$ and suppose that $V$ is a linear representation of $G$ defined over $k$. Let $U \subset V$ be a $G$-equivariant open subset of $V$, also defined over $k$, on which $G$ acts freely, and let $S=V-U$. Then, as long as the codimension $r$ of $S$ in $V$ is sufficiently large, $U$ is an appropriate replacement for $E G$. In fact, we have the following theorem, which is DefinitionProposition 1 of $[3]$.

Theorem 2.1. Let $X$ be an algebraic space with $G$-action, and let $G$ act diagonally on the product $U \times X$. Then the groups

$$
A_{k+|U|-|G|}(U \times X) / G
$$

are independent of $U$ up to isomorphism as long as $r>>0$.

Edidin and Graham define the equivariant Chow group $A_{k}^{G} X$ to be the group $A_{k+|U|-|G|}(U \times X) / G$ of the theorem provided that the codimension of $S$ in $V$ is sufficiently large. The inconvenient aspect of this definition for our purposes is that it only defines the group $A_{k}^{G} X$ as a group up to isomorphism. This is not a serious mathematical problem, but it makes the discussion of maps between equivariant cohomology groups somewhat awkward. Therefore, we intend to replace this definition up to isomorphism by an equivalent definition of $A_{*}^{G} X$ as a limit along the lines of Totaro's definition of $A^{*} B G$ (see Theorem 1.3 of [9]). This new definition will specify the set $A_{*}^{G} X$ uniquely.

2.1. Algebraic Spaces. We need to recall a few results from [3] concerning algebraic spaces. Suppose that $G$ is a linear algebraic group over a field $k$ and $X$ is an algebraic space with a free $G$-action. Let $[X / G]$ be the functor whose sections over an algebraic space $B$ are the principal $G$-bundles $p: E \rightarrow B$ together with an equivariant map $f: E \rightarrow X$. By theorems of Artin, Deligne and Mumford [2], [1], there 
is an algebraic space $Y$ representing the functor $[X / G]$. Moreover, the canonical map $\pi: X \rightarrow Y$ is a principal $G$-bundle. (As a natural transformation of functors, $\pi$ sends a morphism $h: B \rightarrow X$ to the principal $G$-bundle $p: G \times B \rightarrow B$ given by projection on the second factor along with the $G$-equivariant map $f: G \times B \rightarrow X$ given by $f(g, b)=g h(b)$.) The proof of the existence of $Y$ and the fact that $X \rightarrow Y$ is a principal $G$-bundle is sketched in $[3$.

We will use the notation $X_{G}$ to denote the algebraic space $Y$. Note, however, that this notation conflicts with that of [3] where $X_{G}$ is used to denote the space $[U \times X / G]$ for $U$ as in Theorem 2.1.

2.2. Intersection Theory. In [3], Edidin and Graham also show how the intersection theory of Fulton [4] extends to the category of algebraic spaces. In particular, they show that refined Gysin homomorphisms are defined for local complete intersection (l.c.i.) morphisms of algebraic spaces.

Suppose that $f: X \rightarrow Y$ is a map between algebraic spaces with free $G$-actions. Let $f_{G}: X_{G} \rightarrow Y_{G}$ denote the map induced by $f$ on the quotient spaces. Let $P$ be any one of the following properties: proper, flat, smooth, regular embedding, or l.c.i. morphism. Then, if $f$ has property $P$, so does $f_{G}$. The proof is essentially the same as the one used to establish Proposition 2.3.2 of [3]. Namely, note that the morphism $\pi_{Y}: Y \rightarrow Y_{G}$ is faithfully flat and $X \cong X_{G} \times_{Y_{G}} Y$. Thus by descent $f_{G}$ has property $P$ whenever $f$ does.

Similarly, suppose $X$ is an algebraic space with free $G$-action and $p: E \rightarrow X$ is a $G$-equivariant vector bundle morphism. (That is, $E$ is the total space of a $G$-equivariant vector bundle.) Then $p_{G}: E_{G} \rightarrow X_{G}$ is also a $G$-equivariant vector bundle morphism. Again, the proof is identical to that of the analogous result from [3], in this case Lemma 2.4.1: The map $p_{G}: E_{G} \rightarrow X_{G}$ is an affine bundle which is locally trivial in the étale topology because its smooth base change to $X$ is trivial. Since the transition functions are trivial when pulled back to $X$, $p_{G}: E_{G} \rightarrow X_{G}$ is a vector bundle.

2.3. Categorical Substitutes for EG. Let Univ $=k^{\oplus \mathbf{N}}$, a vector space on countably many generators. For any linear algebraic group $G$ over $k$, let $\operatorname{Rep} G$ denote the category of finite-dimensional subspaces $V \subset$ Univ together with homomorphisms $G \rightarrow \mathrm{Gl}(V)$. Morphisms are $G$-equivariant linear maps.

Let $\mathrm{E} G$ be the category whose objects are nonempty open subsets $U \subset V$ for $V$ a representation in $\operatorname{Rep} G$ such that $G$ acts freely on $U$. The morphisms of $\mathrm{E} G$ are $G$-equivariant maps of schemes. Let $\mathrm{E} G_{r}$ denote the full subcategory of $\mathrm{E} G$ consisting of all $U$ such that the complement $S=V-U$ has codimension $\geq r$ in $V$. Remark 1.4 of $\left[9\right.$ shows that $\mathrm{E} G_{r}$ is never empty; that is, for any $r$ we can find a $U \subset V$ such that $V-U$ has codimension $\geq r$ in $V$.

Let FSE $/{ }_{X} G$ denote the category of all algebraic spaces $E$ with free $G$-actions equipped with a smooth morphism $p_{E}: E \rightarrow X$ of fixed relative dimension $n_{E}$. In other words, we assume that the map $p_{E}: E \rightarrow X$ is equidimensional. Morphisms in FSE $/{ }_{X} G$ are $G$-equivariant maps $f: E \rightarrow F$ such that $p_{E}=p_{F} \circ f$.

Now let $i_{X}: \mathrm{E} G \rightarrow \mathrm{FSE} /{ }_{X} G$ be the functor sending $U$ to $U \times X$ with the diagonal $G$-action and with $p_{U \times X}$ given by projection on the second factor. Clearly, if $f$ is a morphism having property $P$ for $P$ any of the properties in subsection [2.2, then $i_{X}(f)=f \times$ id also has property $P$. Moreover, note that any morphism $f: E \rightarrow F$ in FSE / ${ }_{X} G$ is an l.c.i. morphism of codimension $n_{F}-n_{E}$. This is because the graph 
morphism $G_{f}: E \rightarrow E \times_{X} F$ is a regular embedding of codimension $n_{F}$ and the projection $p_{2}: E \times_{X} F \rightarrow F$ is smooth of relative dimension $n_{E}$. It follows then from the results of subsection 2.2 that, for any morphism $f: E \rightarrow F$ in FSE/X $G$, $f_{G}$ is an l.c.i. morphism, also of relative dimension $n_{E}-n_{F}$.

Since $f_{G}$ is an l.c.i. morphism, there is a pullback morphism $f_{G}^{!}: A_{k} F_{G} \rightarrow$ $A_{k+n_{E}-n_{F}} E_{G}$. To simplify the indices that we have to consider, we make the following convention: For $E$ an algebraic space in $\mathrm{FSE} /{ }_{X} G$,

$$
\mathrm{CH}_{k}^{G} E:=A_{k+n_{E}-|G|} E_{G}
$$

where $|G|$ is the dimension of $G$. Then for any morphism $f: E \rightarrow F$ in FSE/X $G$, there is a corresponding Gysin morphism

$$
f_{G}^{!}: \mathrm{CH}_{k}^{G} F \rightarrow \mathrm{CH}_{k}^{G} E .
$$

In other words, $\mathrm{CH}_{k}^{G}$ is a contravariant functor from FSE/X $G$ to the category of abelian groups.

We intend to use the categories $\mathrm{E} G$ and $\mathrm{FSE} /{ }_{X} G$ as substitutes for the topologist's space $E G$. The advantage of $E G$ is that it is a small category and, thus, limits over $\mathrm{E} G$ are well-defined sets. The advantage of FSE/X $G$ is that it is large enough to conveniently hold several important constructions.

If $E$ and $F$ are two spaces in $\mathrm{FSE} /{ }_{X} G$, then $E \times_{X} F$ is naturally a space in FSE / $X$ $G$ if we agree to let $G$ act on the product diagonally. If $U$ is in E $G$ and $E$ is in FSE $/{ }_{X} G$, then $U \times E=i_{X}(U) \times{ }_{X} E$ with the diagonal action is also naturally a space in $\mathrm{FSE} / x G$.

Recall the convention that, if $X$ is an algebraic space, then $|X|$ denotes the maximum of the dimensions of the irreducible components of $X$. We have the following lemma.

Lemma 2.2. Let $U$ be a scheme in $\mathrm{E} G_{r}$ and let $E$ be an algebraic space in $\mathrm{FSE} / X G$. Let $\pi: U \times E \rightarrow E$ be the projection. Then

$$
\pi_{G}^{!}: \mathrm{CH}_{k}^{G} E \rightarrow \mathrm{CH}_{k}^{G} U \times E
$$

is an isomorphism as long as $r>|X|-k$.

Proof. Let $U \subset V$ with $V$ a representation in $\operatorname{Rep} G$, and let $q: V \times E \rightarrow E$ be the projection. This projection induces a vector bundle morphism $q_{G}:(V \times E)_{G} \rightarrow E_{G}$, and it follows that $q_{G}^{*}: A_{j} E_{G} \rightarrow A_{j+|V|}(V \times E)_{G}$ is an isomorphism for all $j$. Therefore $q_{G}^{!}: \mathrm{CH}_{*}^{G} E \rightarrow \mathrm{CH}_{*}^{G} V \times E$ is an isomorphism.

Let $i: U \times E \rightarrow V \times E$ be the inclusion. By our assumption on $U$, the complement $S$ of $U$ in $V$ has codimension $\geq r$. Therefore, $|S \times E| \leq|V|-r+n_{E}+|X|$ and, since $G$ acts freely on $|S \times E|$,

$$
\left|(S \times E)_{G}\right| \leq|V|-r+n_{E}+|X|-|G| .
$$

It follows from the short exact sequence in Chow groups that

$$
i_{G}^{*}: A_{j}(V \times E)_{G} \rightarrow A_{j}(U \times E)_{G}
$$

is an isomorphism as long as

$$
j>|V|-r+n_{E}+|X|-|G| .
$$

Therefore, by (2) and the fact that $n_{U \times E}=|V|+n_{E}$,

$$
i_{G}^{!}: \mathrm{CH}_{k}^{G} V \times E \rightarrow \mathrm{CH}_{k}^{G} U \times E
$$


is an isomorphism whenever

$$
k+|V|+n_{E}-|G|>|V|-r+n_{E}+|X|-|G| .
$$

After cancellation, we see that $i_{G}^{!}: \mathrm{CH}_{k}^{G} V \times E \rightarrow \mathrm{CH}_{k}^{G} U \times E$ is an isomorphism whenever $r>|X|-k$.

Now, since $\mathrm{CH}_{k}^{G}$ is a contravariant functor and $\pi=q \circ i, \pi_{G}^{!}: \mathrm{CH}_{k}^{G} E \rightarrow$ $\mathrm{CH}_{k}^{G} U \times E$ is an isomorphism as long as $r>|X|-k$.

For $U$ and $E$ as above, consider the diagram

$$
U \times X \stackrel{h_{E}}{\longleftarrow} U \times E \stackrel{\pi_{E}}{\longrightarrow} E
$$

where $h_{E}=$ id $\times p_{E}$. By Lemma 2.2 as long as $r>|X|-k$, it makes sense to define a map

$$
t_{U, E}: \mathrm{CH}_{k}^{G} U \times X \rightarrow \mathrm{CH}_{k}^{G} E
$$

by setting $t_{U, E} \alpha=\left(\left(\pi_{E}\right)_{G}^{!}\right)^{-1}\left(h_{E}\right)_{G}^{!} \alpha$. In fact, as we will now see, $t_{U, E}$ is natural in $E$.

Lemma 2.3. Suppose $U$ is in $\mathrm{E} G_{r}$ with $r>|X|-k$ and $f: E \rightarrow F$ is a morphism in $\mathrm{FSE} /{ }_{X} G$. Then, for any $\alpha \in \mathrm{CH}_{k} U \times X$,

$$
f_{G}^{!} t_{U, F}=t_{U, E} .
$$

Proof. The diagram

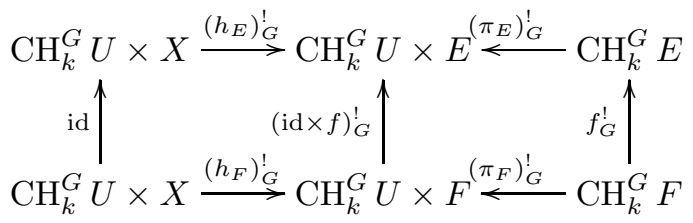

is commutative by the functoriality of $\mathrm{CH}_{k}^{G}$. It is then a simple diagram chase to verify the lemma.

We now state the main theorem of the section.

Theorem 2.4. Suppose $U$ is in $\mathrm{E} G_{r}$. Let $\mathrm{C}$ be any subcategory of $\mathrm{FSE} /{ }_{X} G$ such that

(i) $U \times X$ is an object in $\mathrm{C}$,

(ii) for any object $E$ in $\mathrm{C}, U \times E$ is an object in $\mathrm{C}$,

(iii) the maps $h_{E}: U \times E \rightarrow U \times X$ and $\pi_{E}: U \times E \rightarrow E$ are morphisms in C. Then, for $k>|X|-r$,

$$
\lim _{E \in \mathrm{C}^{\mathrm{OP}}} \mathrm{CH}_{k}^{G} E \cong \mathrm{CH}_{k}^{G} U \times X .
$$

Moreover, the isomorphism $R: \lim _{E \in \mathrm{C}^{\mathrm{op}}} \mathrm{CH}_{k}^{G} E \rightarrow \mathrm{CH}_{k}^{G} U \times X$ can be taken to be the restriction morphism sending an assignment $E \rightsquigarrow \alpha_{E} \in \mathrm{CH}_{k}^{G} E$ to the element $\alpha_{U \times X}$ of $\mathrm{CH}_{k}^{G} U \times X$. 
Proof. By Lemma 2.3 the first statement of the theorem will be proved once we show that for each assignment $E \rightsquigarrow \alpha_{E}$ satisfying the condition that

$$
f_{G}^{!} \alpha_{F}=\alpha_{E} \quad \text { for } f: E \rightarrow F \text { a morphism in C, }
$$

there is a unique $\alpha \in \mathrm{CH}_{k}^{G} U \times X$ such that $\alpha_{E}=t_{U, E} \alpha$. To prove the second statement it then suffices to show that this unique $\alpha$ is in fact $\alpha_{U \times E}$. We will prove both statements simultaneously.

Let $E \rightsquigarrow \alpha_{E}$ be an assignment satisfying condition (10). Then from the diagram

$$
\mathrm{CH}_{k}^{G} U \times X \stackrel{h_{G}^{!}}{\longrightarrow} \mathrm{CH}_{k}^{G} U \times E \stackrel{\pi_{G}^{!}}{\longleftarrow} \mathrm{CH}_{k}^{G} E
$$

we see that $h_{G}^{!} \alpha_{U \times X}=\pi_{G}^{!} \alpha_{E}$; thus,

$$
\alpha_{E}=t_{U, E} \alpha_{U \times X} .
$$

A particular case of (12) is that $\alpha_{U \times X}=t_{U, U \times X} \alpha_{U \times X}$. Suppose then that $\beta$ is any element of $\mathrm{CH}_{k}^{G} U \times X$ such that $t_{U, E} \beta=\alpha_{E}$ for all $E$ in C. Then

$$
t_{U, U \times X}\left(\alpha_{U \times X}-\beta\right)=0 .
$$

However, $t_{U, U \times X}$ is easily seen to be an isomorphism. Thus $\beta=\alpha_{U \times X}$.

Definition 2.5. If $X$ is a $G$-equivariant algebraic space, then set

$$
A_{k}^{G} X=\lim _{U \in \overleftarrow{\mathrm{E}}^{\text {op }}} \mathrm{CH}_{k}^{G} U \times X .
$$

These groups are the G-equivariant Chow groups. By Theorem 2.4, they agree up to isomorphism with the groups defined in [3].

Note that another consequence of Theorem 2.4 is a natural isomorphism

$$
A_{k}^{G} X \cong \lim _{E \in \mathrm{FSE} / \mathrm{X}} G^{\mathrm{op}} \mathrm{CH}_{k}^{G} E .
$$

It will be useful to introduce another category, which can be thought of as lying between E $G$ and FSE $/ x G$. For an algebraic group $G$, let CE $G$ denote the category of all smooth $G$-equivariant spaces with free $G$-actions. Morphisms in CE $G$ are $G$-equivariant morphisms. There is an inclusion of categories $j: \mathrm{E} G \rightarrow \mathrm{CE} G$, and for any $G$-space $X$, a functor $\iota_{X}: \mathrm{CE} G \rightarrow \mathrm{FSE} /{ }_{X} G$ sending $U$ in CE $G$ to $U \times X$ with $G$ acting diagonally. Moreover, the composition $\iota_{x} \circ j=i_{X}$; thus, we have a chain of inclusions

$$
\mathrm{E} G \stackrel{j}{\rightarrow} \mathrm{CE} G \stackrel{l_{X}}{\rightarrow} \mathrm{FSE} /{ }_{X} G .
$$

By Theorem 2.4, (15) induces a chain of isomorphisms

$$
\lim _{E \in \widetilde{F S E}^{-} \times} \mathrm{CH}_{k}^{G} E \cong \lim _{U \leftarrow \widetilde{\mathrm{CE}} G} \mathrm{CH}_{k}^{G} U \times X \cong A_{k}^{G} X .
$$

We will use the isomorphism freely to identify the three groups.

\section{Functorial Properties of Equivariant Intersection Theory}

Following Edidin and Graham ([3], Proposition 2.3.3), we can now show that equivariant intersection theory enjoys essentially the same properties with respect to pushforward and pullback as ordinary non-equivariant intersection theory. We will also show that equivariant intersection has two "change-of-group" maps, restriction and transfer, which are analogous to restriction and transfer in equivariant cohomology. 
3.1. Pushforward and Pullback. Suppose that $f: X \rightarrow Y$ is a proper morphism of $G$-equivariant spaces. Then $f$ induces a proper morphism

$$
\left(\operatorname{id}_{U} \times f\right)_{G}:(U \times X)_{G} \rightarrow(U \times Y)_{G}
$$

for any $U \in \mathrm{E} G$. Thus, from proper pushforward, we obtain morphisms

$$
\left[\left(\mathrm{id}_{U} \times f\right)_{G}\right] *: \mathrm{CH}_{k} U \times X \rightarrow \mathrm{CH}_{k} U \times Y,
$$

and, in the limit over all $U$ in $\mathrm{E} G$, these morphisms induce a map

$$
f_{G *}: A_{k}^{G} X \rightarrow A_{k}^{G} Y,
$$

the equivariant proper pushforward.

3.1.1. The Refined Gysin Homomorphism. Suppose that $f: X \rightarrow Y$ is a $G$ equivariant l.c.i. morphism and that

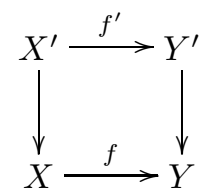

is a pullback diagram with all morphisms $G$-equivariant. Then, given any $U$ in $\mathrm{E} G$, the diagram

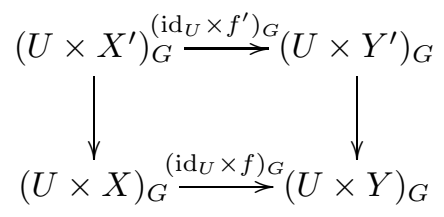

is also a pullback diagram, and $\left(\operatorname{id}_{U} \times f\right)_{G}$ is an l.c.i. morphism by the results of Edidin and Graham quoted in section 2.2. Thus, from Fulton's intersection theory, we obtain a morphism

$$
\left(\operatorname{id}_{U} \times f\right)_{G}^{!}: \mathrm{CH}_{k}^{G} U \times Y^{\prime} \rightarrow \mathrm{CH}_{k-c}^{G} U \times X^{\prime}
$$

where $c$ is the codimension of $f$ as an l.c.i. morphism. Taking the limit over all $U$ in $\mathrm{E} G$, we obtain a morphism

$$
f_{G}^{!}: A_{k}^{G} Y^{\prime} \rightarrow A_{k-c}^{G} X^{\prime}
$$

the equivariant Gysin homomorphism.

Suppose that $\alpha \in A_{k}^{G} Y^{\prime}$, and $E$ is in FSE/X $X^{\prime}$. Using the identification (16), we obtain a class $\left(f_{G}^{!} \alpha\right)_{E} \in \mathrm{CH}_{k-c}^{G} E$. Unfortunately we do not have a description of this class except through the identification (16). However, if $E=U \times X^{\prime}$ for $U \in \mathrm{CE} G$, then we obtain a diagram as in (20), and

$$
\left(f_{G}^{!} \alpha\right)_{U \times X^{\prime}}=\left(\operatorname{id}_{U} \times f\right)_{G}^{!} \alpha_{U \times Y^{\prime}}
$$

where $\alpha_{U \times Y^{\prime}}$ is the class in $\mathrm{CH}_{k}^{G} U \times Y^{\prime}$ induced from $\alpha$ via (16). 
3.2. Chern Classes. If $\pi: V \rightarrow X$ is a $G$-equivariant vector bundle and $E$ is a space in $\mathrm{FSE} /{ }_{X} G$, then $p: V \times_{X} E \rightarrow E$ is also a $G$-equivariant vector bundle with $G$ acting diagonally on the factors. As noted in section [2.2, this implies that $p_{G}:\left(V \times_{X} E\right)_{G} \rightarrow E_{G}$ is a vector bundle. We thus obtain Chern classes $c_{i}\left(V \times{ }_{X} E\right)_{G}$ operating on $A_{*}\left(E_{G}\right)$. If $f: E \rightarrow F$ is a morphism in $\mathrm{FSE} /{ }_{X} G$, then $V \times_{X} E=f^{*}\left(V \times_{X} F\right)$. Thus, $\left(V \times_{X} E\right)_{G}=f_{G}^{*}\left(V \times_{X} F\right)_{G}$. Therefore, if $\alpha \in A_{*}^{G} X$, then

$$
\begin{aligned}
c_{i}\left(V \times_{X} E\right)_{G} \cap \alpha_{E} & =c_{i}\left(V \times_{X} E\right)_{G} \cap f_{G}^{!} \alpha_{F} \\
& =f_{G}^{!}\left(c_{i}\left(V \times_{X} F\right)_{G} \cap \alpha_{F}\right) .
\end{aligned}
$$

We can therefore define the equivariant Chern class $c_{i}^{G}(V)$ to be the operation on $A_{*}^{G} X$ that takes the assignment

$$
E \rightsquigarrow \alpha_{E}
$$

to the assignment

$$
E \rightsquigarrow c_{i}\left(V \times_{X} E\right)_{G} \cap \alpha_{E} .
$$

It is easy to see that this agrees with the definition made by Edidin and Graham in [3].

3.3. Change of Groups. Suppose that $\rho: H \rightarrow G$ is a morphism of linear algebraic groups over $k$. By analogy with equivariant cohomology, we expect a morphism $\rho^{*}: A_{k}^{G} X \rightarrow A_{k}^{H} X$ where $X$ is a $G$-equivariant space and $H$ acts on $X$ through the morphism $\rho$. To define this morphism, we use the categories FSE/ ${ }_{X} H$ and $\mathrm{FSE} /{ }_{x} G$.

For a space $E$ in $\mathrm{FSE} / x H$, let $G \times{ }^{H} E$ denote the quotient of the space $G \times E$ by the action of $H$ given by

$$
h(g, e)=\left(g h^{-1}, h e\right)
$$

where here $h \in H(S), g \in G(S)$ and $e \in E(S)$ for an arbitrary scheme $S$ over $k$. Then $G$ acts on $G \times{ }^{H} E$ on the left by the action: $g_{1}\left(g_{2}, e\right) \mapsto\left(g_{1} g_{2}, e\right)$, and it is easy to see that this action is free. We equip $G \times{ }^{H} E$ with a morphism $p_{G \times{ }^{H} E}: G \times{ }^{H} E \rightarrow X$ given on points $g \in G(S), e \in E(S)$ by $(g, e) \mapsto g p_{E}(e)$. This morphism is well-defined because, for $h \in H(S)$,

$$
p_{G \times{ }^{H} E}\left(g h^{-1}, h e\right)=g h^{-1} p_{E}(h e)=g h^{-1} h p_{E}(e)=g p_{E}(e) .
$$

The morphism $p_{G \times{ }^{H} E}$ is clearly $G$-equivariant, and it is not difficult to check that it is a smooth morphism of relative dimension $n_{E}+|G|-|H|$ where $n_{E}$ is the relative dimension of $p_{E}$. Thus $G \times{ }^{H} E$ is an algebraic space in FSE $/{ }_{G} X$. In fact, the association $E \rightsquigarrow G \times{ }^{H} E$ is the map on objects of a functor $\rho_{+}: \mathrm{FSE} /{ }_{H} X \rightarrow$ FSE $/{ }_{G} X$ defined by setting $\rho_{+}(f)$, for $f: E \rightarrow F$ a morphism in FSE ${ }_{H} X$, equal to the map induced by $\operatorname{id}_{G} \times f$.

Now note that $\left(G \times{ }^{H} E\right)_{G} \cong E_{H}$. To see this, let $\operatorname{pr}_{2}:\left(G \times{ }^{H} E\right) \rightarrow E_{H}$ be the map induced by the projection on the second factor. If $g \in G(S), e \in E(S)$ and $h \in H(S)$ for some base scheme $S$, then $\operatorname{pr}_{2}\left(g h^{-1}, h e\right)=h e$. Thus it is easy to see that $\mathrm{pr}_{2}$ induces a map $p_{2}:\left(G \times{ }^{H} E\right)_{G} \rightarrow E_{H}$. To define an inverse morphism, let inc $_{2}: E \rightarrow\left(G \times{ }^{H} E\right)_{G}$ be the map induced by the inclusion on the second factor. Then $\operatorname{inc}_{2}(h e)=(1, h e)=\left(h^{-1}, e\right)=(1, e)$. Thus inc 2 induces a morphism $i_{2}: E_{H} \rightarrow\left(G \times{ }^{H} E\right)_{G}$. It is then easy to check that $p_{2}$ and $i_{2}$ are inverses. 
3.3.1. Restriction. It is also easy to check that the maps $p_{2}$ and $i_{2}$ are natural in $E$. Thus the functors $E \rightsquigarrow \mathrm{CH}_{k}^{H} E$ and $E \rightsquigarrow \mathrm{CH}_{k}^{G} \rho_{+} E$ are naturally isomorphic. Explicitly, $p_{2}^{*}: C H_{k}^{H} E \rightarrow \mathrm{CH}_{k}^{G} \rho_{+} E$ is an isomorphism with inverse $i_{2}^{*}$. Keeping in mind the natural isomorphism (14), we can thus define the restriction homomorphism $\rho^{*}: A_{k}^{G} X \rightarrow A_{k}^{H} X$ via the composition

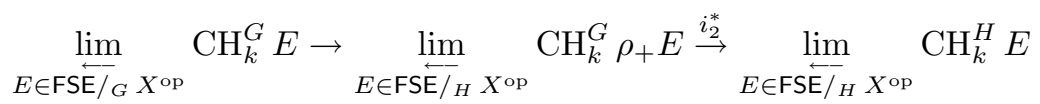

where the first morphism is restriction.

3.3.2. Functoriality of restriction. To show that the restriction homomorphism is functorial, we need to show (a) that $\mathrm{id}^{*}=$ id when id : $G \rightarrow G$ is the identity morphism, and (b) that $(\rho \circ \sigma)^{*}=\sigma^{*} \circ \rho^{*}$ when $\sigma: L \rightarrow H$ and $\rho: H \rightarrow G$ are two group homomorphisms and $X$ is a $G$-space. Before verifying (a) and (b), we prove a lemma.

Lemma 3.1. Let $X$ be a $G$-space, $\rho: H \rightarrow G$ a homomorphism and $E$ a space in $\mathrm{FSE} /{ }_{X} G$. Then the multiplication morphism $m: G \times{ }^{H} E \rightarrow E$ given on points $g \in G(S), e \in E(S)$ by $m(g, e)=g e$ is a morphism in $\mathrm{FSE} /{ }_{X} G$.

Proof. The morphism $m$ is well-defined, because

$$
m\left(g h^{-1}, h e\right)=g e=m(g, e) .
$$

It is clearly $G$-equivariant, and it commutes with the maps to $X$ because

$$
p_{E} \circ m(g, e)=p_{E}(g e)=g p_{E}(e)=p_{G \times{ }^{H}}(g, e) .
$$

To prove statement (a), let $X$ be a $G$-space, $\alpha \in A_{k}^{G} X$, and $E$ a space in FSE $/{ }_{X} G$. Then, according to (25)), $\left(\operatorname{id}^{*} \alpha\right)_{E}=i_{2}^{*} \alpha_{G \times{ }^{G} E}$. Let $m: G \times{ }^{G} E \rightarrow E$ be the morphism of Lemma 3.1. Since it is a morphism in FSE ${ }_{X} G, \alpha_{G \times{ }^{G} E}=m_{G} \alpha_{E}$. Therefore,

$$
\left(\mathrm{id}^{*} \alpha\right)_{E}=i_{2}^{*} m_{G}^{!} \alpha_{E}=\left(m_{G} \circ i_{2}\right)^{!} \alpha_{E} .
$$

Now, let $e \in E(S)$ for a scheme $S$. Then

$$
m_{G} \circ i_{2}(e)=m_{G}(1, e)=e ;
$$

thus, $m_{G} \circ i_{2}=$ id. It follows that $\left(\operatorname{id}^{*} \alpha\right)_{E}=\alpha_{E}$. Thus $\operatorname{id}^{*} \alpha=\alpha$.

(b). To check that $(\rho \circ \sigma)^{*}=\sigma^{*} \circ \rho^{*}$, the important point to note is that the functors $\rho_{+} \circ \sigma_{+}$and $(\rho \circ \sigma)_{+}$are naturally isomorphic.

Explicitly, let

$$
\begin{aligned}
& i_{a}: E_{L} \rightarrow\left(H \times{ }^{L} E\right)_{H}, \quad i_{b}: E_{L} \rightarrow\left(G \times{ }^{L} E\right)_{G}, \\
& i_{c}:\left(H \times{ }^{L} E\right)_{H} \rightarrow\left(G \times{ }^{H}\left(H \times{ }^{L} E\right)\right)_{G}
\end{aligned}
$$

be the isomorphisms induced by inclusion on the second factor. Let

$$
\text { inc }_{13}: G \times{ }^{L} E \rightarrow G \times{ }^{H}\left(H \times{ }^{L} E\right)
$$

be the inclusion on the first and third factors, an isomorphism in $\mathrm{FSE} /{ }_{G} X$; and let $i_{13}=\left(\text { inc }_{13}\right)_{G}:\left(G \times{ }^{L} E\right)_{G} \rightarrow\left(G \times{ }^{H}\left(H \times{ }^{L} E\right)\right)_{G}$ be the induced isomorphism on 
quotient spaces. Then we have a commutative diagram

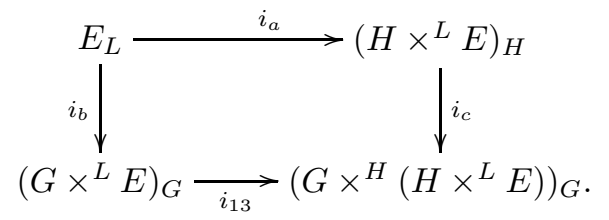

Let $\alpha \in A_{k}^{G} X$ be an equivariant cycle and let $E \in \mathrm{FSE} /{ }_{L} X$. Then, by (25),

$$
\left(\sigma^{*} \rho^{*} \alpha\right)_{E}=i_{a}^{*} i_{c}^{*} \alpha_{G \times{ }^{H}\left(H \times{ }^{L} E\right)}
$$

and

$$
\left((\rho \circ \sigma)^{*} \alpha\right)_{E}=i_{b}^{*} \alpha_{G \times{ }^{L} E} .
$$

However, since inc 13 is a $G$-space morphism,

$$
\alpha_{G \times{ }^{L} E}=i_{13}^{*} \alpha_{G \times{ }^{H}\left(H \times{ }^{L} E\right)} .
$$

Therefore,

$$
\begin{aligned}
\left((\rho \circ \sigma)^{*} \alpha\right)_{E} & =i_{b}^{*} i_{13}^{*} \alpha_{G \times{ }^{H}\left(H \times{ }^{L} E\right)} \\
& =i_{a}^{*} i_{c}^{*} \alpha_{G \times H}\left(H \times{ }^{L} E\right) \\
& =\left(\sigma^{*} \rho^{*} \alpha\right)_{E}
\end{aligned}
$$

by commutativity of diagram (29).

3.4. Transfer. Suppose now that $\rho: H \rightarrow G$ is an inclusion of linear algebraic groups such that the quotient space $G / H$ is proper of dimension $d$. Then, if $X$ is a $G$-space, there is a homomorphism $\operatorname{tr}_{H}^{G}: A_{k}^{H} X \rightarrow A_{k+d}^{G} X\left(\right.$ or $\rho_{*}: A_{k}^{H} X \rightarrow A_{k+d}^{G} X$ ) called the transfer.

To define the transfer, first note that, since $\rho$ is an inclusion, there is a restriction functor $\rho^{+}: \mathrm{FSE} /{ }_{G} X \rightarrow \mathrm{FSE} /{ }_{H} X$ given by viewing a space $E \in \mathrm{FSE} /{ }_{G} X$ as an $H$-equivariant space via $\rho$. If $E \in \mathrm{FSE} /{ }_{G} X$, then the quotient map $q: E_{H} \rightarrow E_{G}$ is proper of relative dimension $d$. Thus proper pushforward induces a map $q_{*}$ : $A_{k} E_{H} \rightarrow A_{k} E_{G}$. Taking into account the re-indexing of the Chow groups of (2), we see that the same proper pushforward induces a map

$$
t_{H}^{G}: \mathrm{CH}_{k}^{H} E \rightarrow \mathrm{CH}_{k+d}^{G} E .
$$

We can thus define the transfer through the composite

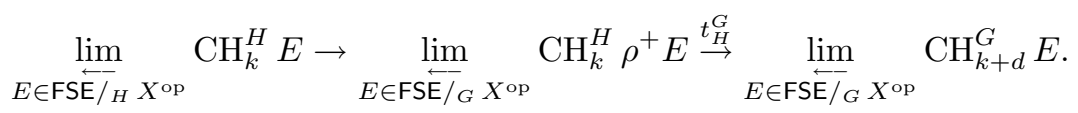

It is an easy exercise to show that the transfer is functorial. That is, if $L \subset H \subset G$ is a sequence of inclusions of groups such that $H / L$ and $G / H$ are both proper, then $\operatorname{tr}_{L}^{G}=\operatorname{tr}_{H}^{G} \circ \operatorname{tr}_{L}^{H}$. 
3.5. Relations between Functors. There are several relations between the functors of proper pushforward, l.c.i. pullback, the restriction homomorphism and transfer. The most important for our purposes is the relation between restriction and transfer when $H \subset G$ is a subgroup of finite index. To deduce this relation, we first prove a more general lemma.

Lemma 3.2. Suppose $\rho: H \rightarrow G$ is an injection, and let $\alpha \in A_{k}^{G} X$ be given by the assignment $E \rightsquigarrow \alpha_{E}$ for $E \in \mathrm{FSE} /{ }_{X} G$. Then, for each $E$, the quotient map $q_{E}: E_{H} \rightarrow E_{G}$ is flat, and the restriction map is given in terms of $q_{E}$ by the equation

$$
\left(\rho^{*} \alpha\right)_{\rho^{+} E}=q_{E}^{*} \alpha_{E}
$$

Proof. The map $q_{E}: E_{H} \rightarrow E_{G}$ is flat because its pullback to $E$ through the faithfully flat morphism $p_{E}: E \rightarrow E_{G}$ is a product. That is, $E_{H} \times_{E_{G}} E \cong G / H \times E$. The map $p_{E}$ is faithfully flat because it is locally a product in the étale topology.

Consider the commutative diagram

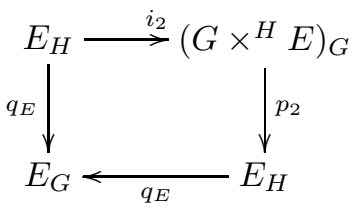

where $i_{2}$ is the isomorphism given by inclusion of the second factor, and $p_{2}$ is its inverse, the projection on the second factor.

By the definition (25),$\left(\rho^{*} \alpha\right)_{\rho^{+} E}=i_{2}^{!} \alpha_{G \times{ }^{H} \rho^{+} E}$. On the other hand, the multiplication map $m: G \times{ }^{H} E \rightarrow E$ of Lemma 3.1 is a morphism in FSE/ ${ }_{X} G$. Thus, since $G \times{ }^{H} E=G \times{ }^{H} \rho^{+} E, \alpha_{G \times{ }^{H} \rho^{+} E}=m^{!} \alpha_{E}$. It thus follows that

$$
\left(\rho^{*} \alpha\right)_{\rho^{+} E}=i_{2}^{!} m ! \alpha_{E} \text {. }
$$

We now claim that $m_{G}=q_{E} \circ p_{2}$. To see this, we compute: $q_{E} \circ p_{2}(g, e)=$ $e(\operatorname{modul} o G)$ while $m_{G}(g, e)=g e(\operatorname{module} G)$; thus, $q_{E} \circ p_{2}(g, e)=m_{G}(g, e)$. Therefore,

$$
\left(\rho^{*} \alpha\right)_{\rho^{+} E}=i_{2}^{!} p_{2}^{*} q_{E}^{*} \alpha_{E}=q_{E}^{*} \alpha_{E}
$$

by the commutativity of (36).

Proposition 3.3. Suppose $X$ is a G-space and $\rho: H \rightarrow G$ is the inclusion morphism of a subgroup of index $N$. Then, for $\alpha \in A_{k}^{H} X, \rho^{*} \operatorname{tr}_{H}^{G} \alpha=N \alpha$.

Proof. Let $\alpha \in A_{k}^{H} X$ be a class given by the assignment $F \rightsquigarrow \alpha_{F}$ with $F \in$ $\mathrm{FSE} /{ }_{H} X$. Then $\operatorname{tr}_{H}^{G} \alpha$ is given by the assignment $E \rightsquigarrow q_{E *} \alpha_{\rho^{+} E}$ for $E$ in FSE $/_{G} X$. It follows then from Lemma 3.2 that, for $E$ in $\mathrm{FSE} / G X$,

$$
\left(\rho^{*} \operatorname{tr}_{H}^{G} \alpha\right)_{\rho^{+} E}=q_{E}^{*}\left(\operatorname{tr}_{H}^{G} \alpha\right)_{E}=q_{E}^{*} q_{E *} \alpha_{\rho^{+} E} .
$$

Since $H$ has index $N$ in $G, q_{E}$ is a degree $N$ morphism. Thus

$$
q_{E}^{*} q_{E *} \alpha_{\rho^{+E}}=N \alpha_{\rho^{+} E} \text {. }
$$

Therefore, $\left(\rho^{*} \operatorname{tr}_{H}^{G} \alpha\right)_{\rho^{+} E}=N \alpha_{\rho^{+} E}$ for all $E$ in $\mathrm{FSE} /{ }_{G} X$.

Now take $E=U \times X$ where $U \in \mathrm{E} G_{r}$ for $r>|X|-k$. After restriction of the $G$-action to $H, U$ is in $\mathrm{E} H_{r}$. Thus

$$
A_{k}^{H} X=\mathrm{CH}_{k}^{H} U \times X=\mathrm{CH}_{k}^{H} \rho^{+} E .
$$


Thus, by (40), $\rho^{*} \operatorname{tr}_{H}^{G} \alpha_{F}=N \alpha_{F}$ for all $F$ in $\mathrm{FSE} /{ }_{H} X$, and the proposition follows.

3.5.1. Restriction and Pullback. Suppose that $f: X \rightarrow Y$ is an 1.c.i. morphism equivariant for the action of a linear algebraic group $G$, and let $\rho: H \rightarrow G$ be a homomorphism of algebraic groups. Then, if

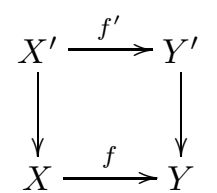

is a $G$-equivariant pullback diagram, the diagram

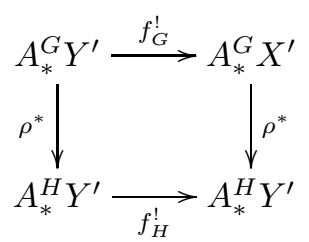

commutes.

To see this, let $\alpha \in A_{*}^{G} Y^{\prime}$ and let $U$ be a space in CE $G$. Then

$$
\left(\rho^{*} f_{G}^{!} \alpha\right)_{U \times X^{\prime}}=i_{2}^{*}\left(f_{G}^{!} \alpha\right)_{G \times{ }^{H}\left(U \times X^{\prime}\right)} .
$$

Now there is an isomorphism

$$
s(X): G \times{ }^{H}\left(U \times X^{\prime}\right) \rightarrow\left(G \times{ }^{H} U\right) \times X^{\prime}
$$

of spaces in $\mathrm{FSE} / X^{\prime} G$ given on the points $g \in G(S), u \in U(S), x \in X^{\prime}(S)$ for $S$ a $k$-scheme by

$$
(g, u, x) \mapsto(g, u, g x)
$$

Thus

$$
\begin{aligned}
\left(\rho^{*} f_{G}^{!} \alpha\right)_{U \times X^{\prime}} & =i_{2}^{*} s\left(X^{\prime}\right)_{G}^{!}\left(f_{G}^{!} \alpha\right)_{\left(G \times{ }^{H} U\right) \times X^{\prime}} \\
& =i_{2}^{*} s\left(X^{\prime}\right)_{G}^{!}(\mathrm{id} \times f)_{G}^{!} \alpha\left(G \times{ }^{H} U\right) \times Y^{\prime}
\end{aligned}
$$

where for the last equation we have used the fact that $G \times{ }^{H} U$ is a space in CE $G$ and thus (23) holds.

On the other hand,

$$
\begin{aligned}
\left(f_{H}^{!} \rho^{*} \alpha\right)_{U \times X^{\prime}} & =(\mathrm{id} \times f)_{H}^{!}\left(\rho^{*} \alpha\right)_{U \times Y^{\prime}} \\
& =(\mathrm{id} \times f)_{H}^{!} i_{2}^{*} \alpha_{G \times H}\left(U \times Y^{\prime}\right) \\
& =(\mathrm{id} \times f)_{H}^{!} i_{2}^{*} s\left(Y^{\prime}\right)_{G}^{!} \alpha_{\left(G \times{ }^{H} U\right) \times Y^{\prime}} .
\end{aligned}
$$

Using the functoriality of the refined Gysin homomorphism (4], Theorem 6.5), we can commute the terms in (46) and (47) to show that (42) commutes.

The commutativity of (42) leads us to define a category of pairs $(G, Y)$ consisting of a linear algebraic group acting on a space $Y$. A morphism $\phi:(H, X) \rightarrow(G, Y)$ in the category of pairs is a pair $(\rho, f)$ consisting of a homomorphism $\rho: H \rightarrow G$ and a map $f: X \rightarrow Y$ such that, for any space $S$ and points $h \in H(S), x \in X(S)$,

$$
f(g x)=\rho(g) f(x) .
$$

We say that $\phi$ is an l.c.i. morphism if $f$ is. 
If $\phi$ is l.c.i., we define $\phi^{!}: A_{*}^{G}(Y) \rightarrow A_{*}^{H}(X)$ by setting

$$
\phi^{!}=f_{H}^{!} \circ \rho^{*}=\rho^{*} \circ f_{G}^{!} .
$$

If $\psi=(\sigma, g):(G, Y) \rightarrow(K, Z)$ is another l.c.i. morphism of pairs, then, using (42), it is easy to see that $(\psi \circ \phi)^{!}=\phi^{!} \circ \psi^{!}$. In fact,

$$
\begin{aligned}
(\psi \circ \phi)^{!} & =(g \circ f)_{H}^{!} \circ(\sigma \circ \rho)^{*} \\
& =f_{H}^{!} \circ g_{H}^{!} \circ \rho^{*} \circ \sigma^{*} \\
& =f_{H}^{!} \circ \rho^{*} \circ g_{G}^{!} \circ \sigma^{*} \\
& =\phi^{!} \circ \psi^{!} .
\end{aligned}
$$

Thus $(G, X) \rightsquigarrow A_{*}^{G}(X)$ is a contravariant functor from the category of pairs with l.c.i. morphisms to the category of abelian groups.

3.6. Transfer and Pullback. If $\rho: H \rightarrow G$ is an inclusion of linear algebraic groups such that $G / H$ is proper of dimension $d$ and $f: X \rightarrow Y$ is an l.c.i.morphism, then it is easy to see that

$$
f_{G}^{!} \circ \operatorname{tr}_{H}^{G}=\operatorname{tr}_{H}^{G} \circ f_{H}^{!} .
$$

This follows from the fact that pullback through an l.c.i. morphism commutes with pushforward through a proper morphism (4], Theorem 6.2 (a) and Proposition $1.7)$.

3.6.1. Restriction and Chern Classes. Let $\rho: H \rightarrow G$ be a homomorphism of linear algebraic groups, and let $V$ be a $G$-equivariant vector bundle over $X$. Let $\rho^{*}(V)$ denote $V$ viewed as an $H$-equivariant bundle through the homomorphism $\rho$. Then, for $\alpha \in A_{*}^{G} X$,

$$
\rho^{*}\left(c_{i}^{G}(V) \cap \alpha\right)=c_{i}^{H} \rho^{*}(V) \cap \alpha .
$$

The proof of this is similar to the proof of the commutativity of (42).

3.7. Automorphisms. An automorphism of a pair $(G, X)$ is a morphism $\phi=$ $(\rho, f):(G, X) \rightarrow(G, X)$ that has an inverse. We say that $\phi$ is inner if there is an $h \in G(k)$ such that, for any $k$-space $S$ and any two $S$-valued points $g \in G(S)$ and $x \in X(S)$,

$$
\rho(g)=h_{S} g h_{S}^{-1}, \quad f(x)=h_{S} x .
$$

Note that inner automorphisms are always l.c.i. morphisms.

Proposition 3.4. If $\phi:(G, X) \rightarrow(G, X)$ is an inner automorphism of a pair $(G, X)$, then $\phi^{!}: A_{k}^{G} X \rightarrow A_{k}^{G} X$ is the identity.

Proof. It suffices to show that $\left(\phi^{!} \alpha\right)_{U}=\alpha_{U}$ for any $\alpha \in A_{k}^{G} X$ and any $U \in \mathrm{E} G$. Let $\phi=(\rho, f)$ and let $h \in G(k)$ be the group element such that $f$ is multiplication by $h$. Let $G \times{ }^{G} U$ denote the quotient of $G \times U$ by the diagonal action given on $S$-points by

$$
g_{1}\left(g_{2}, u\right)=\left(g_{2} h_{S} g_{1}^{-1} h_{S}^{-1}, g_{1} u\right)
$$


for $g_{1}, g_{2} \in G(S)$. Let $i_{2}: U_{G} \rightarrow\left(G \times{ }^{G} U\right)_{G}$ denote the isomorphism induced by inclusion on the second factor. Then, since $\phi^{!}=\rho^{*} \circ f^{!}$, we have $\left(\phi^{!} \alpha\right)_{U}=$ $i_{2}^{!}\left(\left(f^{!} \alpha\right)_{G \times{ }^{G} U}\right)$. Pulling out the $f$, we have

$$
\left(\phi^{!} \alpha\right)_{U}=f_{G}^{!} i_{2}^{!}\left(\alpha_{G \times G}\right)
$$

To prove the proposition, we want to compute $\alpha_{G \times{ }^{G} U}$ in terms of $\alpha_{U}$. To do this, we define a $G$-equivariant morphism $l: G \times{ }^{G} U \rightarrow U$ given on scheme-theoretic points $g \in G(S), u \in U(S)$ by

$$
l(g, u)=g h_{S} u
$$

Since $G$ acts on the right on $G \times{ }^{G} U$, it is obvious that $l$ is $G$-equivariant provided that it is well-defined. That this is so can be easily checked using (53).

Since $l$ is $G$-equivariant, $\alpha_{G \times{ }^{G} U}=l_{G}^{!} \alpha_{U}$. We thus have

$$
\left(\phi^{!} \alpha\right)_{U}=f_{G}^{!} i_{2}^{!} l_{G}^{!} \alpha_{U} .
$$

Now consider the composition $r$ in the diagram

$$
U \times X \stackrel{\text { id } \times f}{\rightarrow} U \times X \stackrel{i_{2} \times \text { id }}{\longrightarrow}\left(G \times{ }^{G} U\right) \times X \stackrel{l \times \text { id }}{\longrightarrow} U \times X .
$$

For $u \in U(S), x \in X(S)$ two $S$-valued points, $r$ is given by $(u, x) \mapsto\left(h_{S} u, h_{S} x\right)$. Thus $r_{G}=$ id. It follows that

$$
f_{G}^{!} i_{2}^{!} l_{G}^{!} \alpha_{U}=r_{G}^{!} \alpha_{U}=\alpha_{U}
$$

\section{Equivariant Cycle Class}

In this section, a few general results on Chow groups necessary for the construction of $S$. are collected. There are two main goals: (i) to explain why equivariant cycles give classes in equivariant Chow groups (this fact is mentioned in the proof of [3], Proposition 1), and (ii) to show that two equivariant cycles are equivalent if they belong to the same equivariant rational family.

Definition 4.1. The group $Z_{k}\left(X \mid \mathbf{P}^{1} ; \Lambda\right)$ is the subgroup of $(k+1)$-dimensional cycles $\sum_{i} \lambda_{i}\left[V_{i}\right]$ in $X \times \mathbf{P}^{1}$ such that all $V_{i}$ map dominantly to $\mathbf{P}^{1}$.

Following the conventions of (1.2), the ring $\Lambda$ will be suppressed in the notation, and the group of $\Lambda$-cycles $Z_{k}(X ; \Lambda)=Z_{k} X \otimes \Lambda$ will be written as $Z_{k} X$.

Following the notation in [4, for $P \in \mathbf{P}^{1}$ and $V$ a variety mapping dominantly to $\mathbf{P}^{1}$, let $V(P)$ denote the fiber above $P$. The definition is extended to $Z_{*}\left(X \mid \mathbf{P}^{1}\right)$ by linearity.

Both $Z_{k}(-)$ and $Z_{k}\left({ }_{-} \mid \mathbf{P}^{1}\right)$ can be viewed as presheaves in the étale topology. That $Z_{k}(-)$ is actually a sheaf in the étale topology is proved in [5] (page 211) for $\Lambda=\mathbf{Z}$. The argument is easily extended to the case of an arbitrary ring $\Lambda$. It is also easily extended to show that $Z_{k}\left(-\mid \mathbf{P}^{1}\right)$ is a sheaf.

Proposition 4.2. Two cycles $\alpha_{0}$ and $\alpha_{\infty}$ in $Z_{k} X$ are rationally equivalent $\Leftrightarrow$ there is a cycle $\beta \in Z_{k}\left(X \mid \mathbf{P}^{1}\right)$ with $\beta(0)=\alpha_{0}$ and $\beta(\infty)=\alpha_{\infty}$.

Proof. First consider the case $\Lambda=\mathbf{Z}$. By [4, Example 1.6.2, $\alpha_{0}$ and $\alpha_{\infty}$ are equivalent if and only if there are a positive cycle $Z \in Z_{k}\left(X \mid \mathbf{P}^{1}\right)$ and a positive cycle $\gamma$ on $X$ with

$$
Z(0)=\alpha_{0}+\gamma, \quad Z(\infty)=\alpha_{\infty}+\gamma
$$


To prove $(\Rightarrow)$, set $\beta=Z-\gamma \times\left[\mathbf{P}^{1}\right]$. To prove $(\Leftarrow)$, write $\beta$ as $\beta^{+}-\beta^{-}$where each term is either positive or 0 . Then, setting $Z=\beta^{+}$shows that $\alpha_{0}+\beta^{-}(0) \sim$ $\alpha_{\infty}+\beta^{-}(\infty)$. This shows that $\alpha_{0}$ and $\alpha_{\infty}$ are equivalent.

For arbitrary $\Lambda$, the proposition is equivalent to the statement that the sequence

$$
Z_{k}\left(X \mid \mathbf{P}^{1}\right) \rightarrow Z_{k} X \rightarrow A_{k} X \rightarrow 0
$$

is exact with the first map given by $\beta \mapsto \beta(\infty)-\beta(0)$. Thus, since the proposition holds for $\Lambda=\mathbf{Z}$, it holds for arbitrary $\Lambda$ by the right exactness of $\otimes$.

Suppose a linear algebraic group $G$ acts on $X$ with action map $a: G \times X \rightarrow X$. Let $p_{2}: G \times X \rightarrow X$ be the projection. Note that both $p_{2}$ and the action map are automatically flat morphisms.

4.3. If $F$ is a $G$-equivariant presheaf of abelian groups in the étale topology, then $a^{*} F=p_{2}^{*} F$, and the fixed sections of $F$ are defined as

$$
F^{G}=\operatorname{ker}\left(F(X) \stackrel{a^{*}-p_{2}^{*}}{\rightarrow} F(G \times X)\right) .
$$

In particular, we can consider the groups $Z_{*}(X)^{G}$ and $Z_{*}\left(X \mid \mathbf{P}^{1}\right)^{G}$. Now assume that $G$ acts freely on $X$. Then there is a quotient $Y$. Moreover, the quotient morphism $\pi: X \rightarrow Y$ is flat. Thus we have flat pullback maps $\pi^{*}: Z_{k}(Y) \rightarrow$ $Z_{k}(X)^{G}$ and $\pi^{*}: Z_{*}\left(Y \mid \mathbf{P}^{1}\right) \rightarrow Z_{*}\left(X \mid \mathbf{P}^{1}\right)^{G}$.

Now assume that $G$ is finite and étale as a group scheme over the base field. It follows then that the quotient $\pi: X \rightarrow Y$ is an étale morphism. This implies that $\pi^{*}: Z_{k} Y \rightarrow Z_{k}(X)^{G}$ and $\pi^{*}: Z_{k}\left(Y \mid \mathbf{P}^{1}\right) \rightarrow Z_{k}\left(X \mid \mathbf{P}^{1}\right)^{G}$ are in fact isomorphisms. When $\Lambda=\mathbf{Z}$, we can easily give the inverse map. Note that if $G$ is finite and étale, $\pi_{*} \pi^{*}$ is multiplication by the order $\# G$. Thus $\frac{1}{\# G} \pi_{*}$ restricted to $\left(Z_{k} X\right)^{G}$ (resp. to $\left.Z_{k}\left(X \mid \mathbf{P}^{1}\right)^{G}\right)$ is inverse to $\pi^{*}$.

Proposition 4.4. Let $G$ be a finite, étale group scheme acting freely on $X$. Two cycles $\alpha_{0}$ and $\alpha_{\infty}$ in $\left(Z_{*} X\right)^{G}$ determine the same class in $A_{*}(X / G)$ if there is a cycle $\beta \in \mathbf{Z}_{*}\left(X \mid \mathbf{P}^{1}\right)^{G}$ such that $\beta(0)=\alpha_{0}$ and $\beta(\infty)=\alpha_{\infty}$

Proof. This is simply a matter of noticing that the diagram

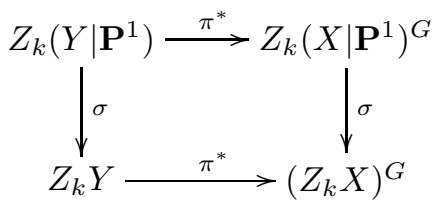

commutes when $\sigma$ is either $\beta \mapsto \beta(0)$ or $\beta \mapsto \beta(\infty)$.

4.5. Now assume that $G$ acts properly, but not necessarily freely, on $X$. Let $\alpha$ be a cycle in $\left(Z_{k} X\right)^{G}$ (resp. $\left.Z_{k}\left(X \mid \mathbf{P}^{1}\right)^{G}\right)$. For any $U \in E G$ of dimension $d, \alpha \mapsto[U] \times \alpha$ gives a map $\left(Z_{k} X\right)^{G} \rightarrow Z_{k+d}(U \times X)^{G}\left(\right.$ resp. $\left.Z_{k}\left(X \mid \mathbf{P}^{1}\right)^{G} \rightarrow Z_{k+d}\left(U \times X \mid \mathbf{P}^{1}\right)^{G}\right)$. But, since the action of $G$ on $U \times X$ is free, $\left(Z_{*} U \times X\right)^{G} \cong Z_{*}\left((U \times X)_{G}\right)$ (resp. $\left.\left(Z_{*} U \times X \mid \mathbf{P}^{1}\right)^{G} \cong Z_{*}\left((U \times X)_{G} \mid \mathbf{P}^{1}\right)\right)$. Thus, for every $\alpha \in Z_{k}(X)^{G}$, we have a class $\left(\pi^{*}\right)^{-1}([U] \times \alpha) \in Z_{k+d}(U \times X)_{G}$. In the limit, these classes give a cycle class $\mathrm{cl}^{G}(\alpha) \in A_{k}^{G} X$.

4.6. It follows from Proposition 4.4 that if $\beta \in Z_{k}\left(X \mid \mathbf{P}^{1}\right)^{G}$, then $[U] \times \beta(0)$ and $[U] \times \beta(\infty)$ represent the same class in $A_{k+d}(X \times U)_{G}$ and thus in $A_{k}^{G} X$. This implies that two equivariant cycles $\alpha_{0}$ and $\alpha_{\infty}$ in $\left(Z_{k} X\right)^{G}$ have the same $G$-equivariant cycle 
class if there is a cycle $\beta \in Z_{k}\left(X \mid \mathbf{P}^{1}\right)^{G}$ with $\beta(0)=\alpha_{0}$ and $\beta(\infty)=\alpha_{\infty}$. In other words, equivariant rational equivalences induce equivalences in $A_{*}^{G} X$.

We will need the following basic proposition to prove that Steenrod operations are additive.

Proposition 4.7. Let $G$ be a finite, constant group scheme over $k$. If $Z \in Z_{k} X$, then

$$
\operatorname{tr}_{1}^{G} \operatorname{cl}^{1}(Z)=\mathrm{cl}^{G}\left(\sum_{g \in G}[g Z]\right)
$$

Here we write 1 for the trivial group.

Proof. Let $U$ be any object in $\mathrm{E} G$. Since $G$ is finite and constant, it is étale. Thus the map $\pi: U \times X \rightarrow(U \times X)_{G}$ is étale and induces an isomorphism

$$
\pi^{*}: Z_{*}(U \times X)_{G} \rightarrow\left(Z_{*}(U \times X)\right)^{G} .
$$

By definition $\left(\operatorname{tr}_{1}^{G} \operatorname{cl}^{1}(Z)\right)_{U \times X}$ is the class in $\mathrm{CH}_{k}^{G}(U \times X)$ of

$$
\pi_{*}[U \times Z] \in Z_{k+|U|}(U \times X)_{G} .
$$

The right-hand side of (57) is the class in $\mathrm{CH}_{k}^{G}(U \times X)$ of

$$
\left(\pi^{*}\right)^{-1} \sum_{g \in G}[U \times g Z] \in Z_{k+|U|}(U \times X)_{G} .
$$

The proposition follows by applying $\pi^{*}$ to (58) and (59) and noting the equality of the results.

Remark 4.8. The proof above uses the fact that the result of applying $\pi^{*}$ to (58) is equal to the result of applying $\pi^{*}$ to (59) before passing to rational equivalence. In fact, the proof makes essential use of the fact that $\pi^{*}: Z_{k} Y \rightarrow\left(Z_{k} X\right)^{G}$ is an isomorphism when $Y=X / G$ for $G$ a finite, étale group scheme acting freely on $X$. However, it is not in general true that $\pi^{*}: A_{k} Y \rightarrow\left(A_{k} X\right)^{G}$ is an isomorphism in this situation. For example, if $E$ is an elliptic curve over $\mathbf{C}$ and $G$ is the group of 2-torsion points, then $E / G=E$ with $\pi: E \rightarrow E$ represented by the multiplicationby-two map. If we take $\Lambda=\mathbf{Z} / 2$, then $\pi^{*}: A_{0} E \rightarrow\left(A_{0} E\right)^{G}$ is trivial.

We also remark that, by the obvious identification of $A_{k}^{1} X$ with $A_{k} X, \operatorname{cl}^{1}(Z)$ is simply the cycle class $[Z] \in A_{k} X$.

\section{The Fundamental Operation}

Let $S(n)$ denote the symmetric group on $n$ letters and $C(n)$ denote the cyclic group with $n$ elements, viewed as a subgroup in the obvious way. Let $\tilde{R}$ denote the standard $S(n)$-representations on $k^{n}$ and let $R$ denote the reduced regular representation, i.e., the cokernel of the map $\mathbf{1} \rightarrow \tilde{R}$ from the trivial representation. One can view these representations as bundles over $B S(n)$.

For a cycle $\alpha \in Z_{k} X$ let $\alpha^{\times n} \in Z_{n k} X^{n}$ denote its $n$-fold exterior product with itself ([4], 1.10). Since $\alpha^{\times n}$ is $S(n)$-invariant, it defines a class $\left[\alpha^{\times n}\right] \in\left(Z_{n k} X^{n}\right)^{G}$ for any $G \leq S(n)$. 
5.1. If $[V] \in Z_{i}\left(X \mid \mathbf{P}^{1}\right)$ and $[W] \in Z_{j}\left(Y \mid \mathbf{P}^{1}\right)$ are two classes corresponding to subvarieties $V$ and $W$, then $V$ and $W$ are both flat over $\mathbf{P}^{1}$. This implies that $V \times \mathbf{P}^{1} W$ is flat over $\mathbf{P}^{1}$. Thus every component of $V \times \mathbf{P}^{1} W$ maps dominantly to $\mathbf{P}^{1}$. Consequently $\left[V \times{ }_{\mathbf{P}^{1}} W\right] \in Z_{i+j}\left(X \times Y \mid \mathbf{P}^{1}\right)$, and linearity gives a product

$$
Z_{i}\left(X \mid \mathbf{P}^{1}\right) \otimes Z_{j}\left(Y \mid \mathbf{P}^{1}\right) \rightarrow Z_{i+j}\left(X \times Y \mid \mathbf{P}^{1}\right),
$$

written $\beta \otimes \gamma \mapsto \beta \times \times_{\mathbf{P}^{1}} \gamma$. In particular, any $\beta \in Z_{k}\left(X \mid \mathbf{P}^{1}\right)$ can be crossed with itself to obtain a class $\beta_{\mathbf{P}^{1}}^{\times n} \in Z_{n k}\left(X^{n} \mid \mathbf{P}^{1}\right)$.

Proposition 5.2. The map $\alpha \mapsto \alpha^{\times n}$ factors through rational equivalence to give a map $P_{G}^{n}: A_{k} X \rightarrow A_{n k}^{G} X^{n}$ for any $G \leq S(n)$.

Proof. Two cycles $\alpha_{0}$ and $\alpha_{\infty}$ are equivalent iff there a cycle $\beta \in \mathbf{Z}_{k}\left(X \mid \mathbf{P}^{1}\right)$ with $\beta(0)=\alpha_{0}$ and $\beta_{\infty}=\alpha_{\infty}$. In this case, $\beta_{\mathbf{P}^{1}}^{\times n}$ is a cycle in $Z_{n k}\left(X^{n} \mid \mathbf{P}^{1}\right)^{G}$, with $\beta_{\mathbf{P}^{1}}^{\times n}(0)=\alpha_{0}^{\times n}$ and $\beta_{\mathbf{P}^{1}}^{\times n}(\infty)=\alpha_{\infty}^{\times n}$.

Proposition 5.3. Consider a morphism $f: X \rightarrow Y$ and a cycle $\alpha \in A_{k} Y$. Let $f_{G}^{\times n}$ denote the obvious $G$-equivariant morphism from $X_{G}^{n}$ to $Y_{G}^{n}$.

(i) For $f$ proper, $f_{G}^{\times n}$ is also proper, and $\left(f_{G}^{\times n}\right)_{*} P_{G}^{n}(\alpha)=P_{G}^{n}\left(f_{*} \alpha\right)$.

(ii) For $f$ flat, $f_{G}^{\times n}$ is also flat, and $\left(f_{G}^{\times n}\right)^{*} P_{G}^{n}(\alpha)=P_{G}^{n}\left(f^{*} \alpha\right)$.

(iii) For $f$ a regular embedding (resp. l.c.i. morphism), $f_{G}^{\times n}$ is also a regular embedding (resp. lci. morphism), and $\left(f_{G}^{\times n}\right)^{!} P_{G}^{n}(\alpha)=P_{G}^{n}\left(f^{!} \alpha\right)$. (Here $f^{!}$ is Fulton's refined Gysin homomorphism [4.)

Proof. (i) is easy, since it is true on the level of cycles, that is, in $Z_{n k}\left(X^{n}\right)$. (ii): If $f$ is flat, then it is easy to see that $f^{\times n}$ is also. Thus $f_{G}^{\times n}$ is also flat by the results of [3] recalled in section 2.2 It is easy to see that the required commutativity actually holds on the level of cycles.

Once (iii) is proved for regular embeddings, the statement for l.c.i. morphisms will be a consequence of (ii). So assume $f$ is a regular embedding of codimension $d$. Let $N$ be the normal bundle, and let

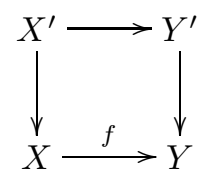

be a pullback diagram. Let $N^{\prime}=N_{\mid X^{\prime}}$.

The refined Gysin homomorphism of [4, $f^{!}: A_{k} Y^{\prime} \rightarrow A_{k-d} X^{\prime}$, is constructed as the composition of the specialization homomorphism $\sigma: A_{k} Y^{\prime} \rightarrow A_{k} N^{\prime}$ with the isomorphism $\left(\pi^{*}\right)^{-1}: A_{k} N^{\prime} \rightarrow A_{k-d} X^{\prime}$.

The map $\sigma$ is defined on the level of cycles by the rule $\sigma[V]=\left[C_{V \cap X} V\right]$. (Here $\left[C_{V \cap X} V\right]$ is the normal cone of $V \cap X$ in $V$ - a subscheme of $N^{\prime}$.) One then sees that $\sigma$ commutes with $P_{G}^{n}$ on the level of cycles, i.e., the diagram

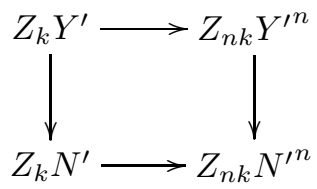

commutes. 
On the other hand, the isomorphism $\pi^{*}: A_{k-d} X^{\prime} \rightarrow A_{k} N^{\prime}$ is simply a flat pullback. Thus the diagram

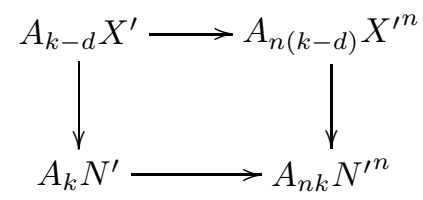

also commutes by (ii). The result then follows by splicing the two diagrams together.

If $V \rightarrow X$ is a $G$-equivariant vector bundle of rank $r$, let $P_{G}^{n}(V)$ denote the product of $n$ copies of $V$ over $n$ copies of $X$ as a $G$-equivariant bundle.

Corollary 5.4. Let $\alpha \in A_{*} X$ and let $c_{\mathrm{top}}(V)$ denote the top Chern class $c_{r}(V)$ of $V$, that is, the top Chern class of $V$. Then $P_{G}^{n}\left(\alpha \cap c_{\mathrm{top}}(V)\right)=P_{G}^{n}(\alpha) \cap c_{\mathrm{top}}\left(P_{G}^{n} V\right)$.

Proof. Let $i: X \rightarrow V$ be the inclusion of the 0 -section. Then $\alpha \cap c_{\text {top }}(V)=i^{!} i_{*} \alpha$. The corollary then follows from the successive application of (i) and (iii) of the previous proposition.

\section{Construction}

Consider a pair $(W, X)$ where $X \rightarrow W$ is an embedding of $X$ into a smooth connected algebraic space $W$ of dimension $d$. Set $m=(n-1) d$, and form the $S(n)$-equivariant pullback diagram

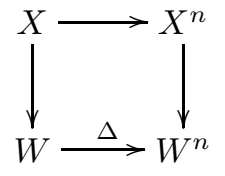

where $S(n)$ is acting on $W^{n}$ and on $X^{n}$ by permuting the factors. We let $S(n)$ act trivially on $W$ and on $X$, and we take $\Delta$ to be the diagonal embedding.

Definition 6.1. For $G \leq S(n), d_{G}^{W}: A_{k}^{G} X^{n} \rightarrow A_{k-m}^{G} X$ is the map given by $d_{G}^{W}(\alpha)=\Delta_{G}^{!} \alpha$. We will write $D_{G}^{W}$ for $d_{G}^{W} \circ P_{G}^{n}$.

Remark 6.2. One way to keep track of the degrees in the definition is to re-index the groups. For a pair $(W, X)$, define $A_{G}^{k}[W, X]=A_{d-k}^{G} X$. Then the re-indexed $\operatorname{map} d_{G}^{W}: A_{G}^{k}\left[W^{n}, X^{n}\right] \rightarrow A_{G}^{k}[W, X]$ preserves the degree, and $D_{G}^{W}$ maps $A^{k}[W, X]$ to $A_{G}^{n k}[W, X]$. This is useful for proving the Adem relations, but for most of this paper we prefer to keep the usual grading.

The next proposition allows us to control the dependence of $d_{G}^{W}$ on the smooth space $W$. Eventually, we will be able to use it to "factor out" the $W$-dependence from our definition of the Steenrod operation $S_{\bullet}$.

Proposition 6.3. Let $j_{i}: X \rightarrow W_{i}$ be two embeddings of $X$ into two smooth spaces $W_{i}$. Then

$$
c_{\text {top }}\left(\left.R \otimes T W_{2}\right|_{X}\right) \cap d_{G}^{W_{1}}=c_{\text {top }}\left(\left.R \otimes T W_{1}\right|_{X}\right) \cap d_{G}^{W_{2}} .
$$


Proof. For $i=1,2$, we have diagrams

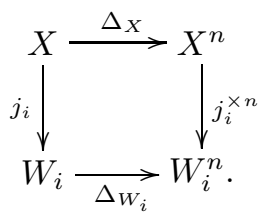

Since $\left.R \otimes T W_{i}\right|_{X}=j_{i}^{*} N_{W_{i}} W_{i}^{\times n}$, we need to show that

$$
c_{\mathrm{top}} j_{1}^{*} N_{W_{1}} W_{1}^{\times n} \cap \Delta_{W_{2}}^{!}=c_{\mathrm{top}} j_{2}^{*} N_{W_{2}} W_{2}^{\times n} \cap \Delta_{W_{1}}^{!} .
$$

This equality is a special case of the following lemma.

Lemma 6.4. Let $X$ and $Y$ be two $G$-equivariant spaces and, for $i=1,2$, let

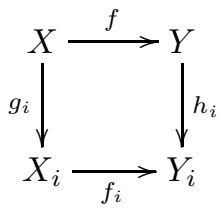

be $G$-equivariant pullback diagrams with the maps $f_{i}: X_{i} \rightarrow Y_{i}$ regular embeddings. Let $N_{i}=g_{i}^{*} N_{X_{i}} Y_{i}$, and let $n_{i}=$ rk $N_{i}$. Then

$$
c_{n_{1}} N_{1} \cap f_{2}^{!} \alpha=c_{n_{2}} N_{2} \cap f_{1}^{!} \alpha
$$

for any class $\alpha \in A_{*}^{G} Y$.

Proof. The proof uses the notion of an excess normal bundle and Fulton's Excess Intersection formula (4], Theorem 6.3).

Form the diagram

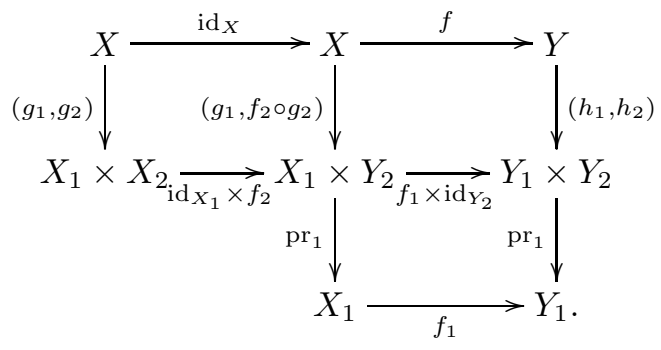

The squares in (64) are pullbacks. For the two squares on the right side, this is automatic. For the one on the left, we need to use the fact that $f$ is an embedding, and this follows from the assumption that the $f_{i}$ are embeddings.

Now let $\alpha$ be a class in $A_{*}^{G} Y$. Since $f_{1} \times \mathrm{id}_{Y_{2}}$ and $f_{1}$ are both regular embeddings of the same codimension (namely $n_{1}$ ), the excess intersection bundle for the right two squares is trivial. Thus

$$
\left(f_{1} \times \operatorname{id}_{Y_{2}}\right)^{!} \alpha=f_{1}^{!} \alpha
$$


On the other hand, note that the left-hand side of (64) fits into a larger couple of pullback diagrams

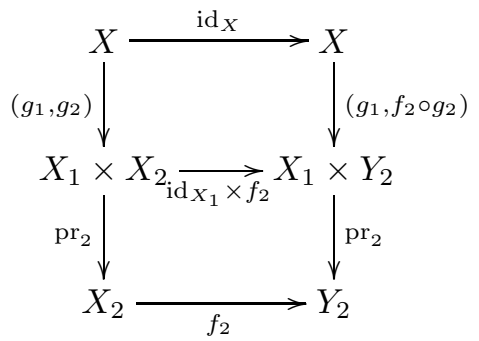

where again the excess intersection bundle for the bottom square is trivial. Moreover, the top horizontal arrow of (66) $\mathrm{id}_{X}$, is a regular embedding. Thus, for $\beta \in A_{*}(X)$,

$$
\left(\operatorname{id}_{X_{1}} \times f_{2}\right)^{!} \beta=f_{2}^{!} \beta=c_{n_{2}} N_{2} \cap \beta .
$$

The last equality follows from the excess intersection formula applied to the top and bottom rows of the diagram.

Finally, consider the diagram

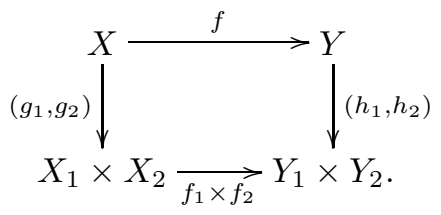

This is the composition of the top two squares of (64); thus,

$$
\begin{aligned}
\left(f_{1} \times f_{2}\right)^{!} \alpha & =\left(\operatorname{id}_{X_{1}} \times f_{2}\right)^{!}\left(f_{1} \times \operatorname{id}_{Y_{2}}\right)^{!} \alpha \\
& =c_{n_{2}} N_{2} \cap f_{1}^{!} \alpha .
\end{aligned}
$$

The first equality follows from the functoriality theorem for the refined Gysin (Theorem 6.5 of [4]), and the second follows by combining equations (65) and (67).

Now we can interchange the roles of $f_{1}$ and $f_{2}$ in the proof of equation (69), and, when we do, we see that

$$
\left(f_{1} \times f_{2}\right)^{!} \alpha=c_{n_{1}} N_{1} \cap f_{2}^{!} \alpha .
$$

Thus we obtain the desired result, equation (63), by equating the right-hand sides of equations (69) and (70).

Proposition 6.5. Let

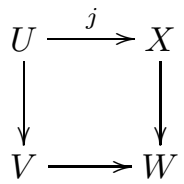

be a commutative diagram of injective maps with the horizontal maps open embeddings and with $W$ (and thus $V$ ) smooth.

(i) For $\alpha \in A_{*}^{G} X^{n}, j^{\times n *}\left(d_{G}^{W} \alpha\right)=d_{G}^{V}\left(j^{\times n *} \alpha\right)$.

(ii) For $\alpha \in A_{*} X, j^{*}\left(D_{G}^{W} \alpha\right)=D_{G}^{V}\left(j^{*} \alpha\right)$. 
Proof. (i) implies (ii) by Proposition [5.3, In the case $V=W$, (i) follows from Theorem 6.2 (a) of [4] - the commutativity of flat pullback with the refined Gysin. The general case then follows from Theorem 6.3 of [4] (the Excess Intersection Formula).

\section{Chow Theory of Cyclic Groups}

Let $n$ be an integer not divisible by char $(k)$, and let $\mu_{n}$ be the linear algebraic group of $n$-th roots of unity. (As an algebraic variety, this is $\operatorname{Spec} k[t] /\left(t^{n}-1\right)$.) The canonical one-dimensional representation of $\mu_{n}$ gives rise to an equivariant line bundle $L$ over $\mathrm{pt}_{k}$. Set $l=c_{1}(L) \in A^{1} B \mu_{n}$. The following result is given in 9] for $k=\mathbf{C}$. Here we give a proof of the (certainly well-known) general case.

Theorem 7.1. (i) $A^{*} B \mu_{n}=\Lambda[l] /(n l)$. (ii) If $X$ is an algebraic space with trivial $\mu_{n}$-action, then $A_{*}^{\mu_{n}} X=A_{*} X \otimes A^{*} B \mu_{n}$.

Proof. Let $E=\mathbf{A}^{r+1}-\{0\}$ with the diagonal action of $\mu_{n}$. Write $B=E / \mu_{n}$, and note that $\mathbf{P}^{r}=E / \mathbf{G}_{m}$. Now let $\mathbf{G}_{m}$ act on $\mathbf{A}^{1} \times E$ on the right by the formula $(a, x) \lambda=\left(\lambda^{n} a, \lambda x\right)$. The resulting quotient $\left(\mathbf{A}^{1} \times E\right) / \mathbf{G}_{m}$ is then the total space of the line bundle $\mathcal{O}(n)$ on $\mathbf{P}^{r}$. The space $B$ embeds as an open subset of $\mathcal{O}(n)$ via the map $j: e \mapsto(1, e)$. The complement of $j(B)$ is the 0 -section, which we will simply write as $\mathbf{P}^{r}$. For any $X$ we then have an exact sequence of groups

$$
A_{i}\left(\mathbf{P}^{r} \times X\right) \rightarrow A_{i}(\mathcal{O}(n) \times X) \rightarrow A_{i}(B \times X) \rightarrow 0 .
$$

For $X=\mathrm{pt}$ the theorem follows from the elementary computation of the map on the left-hand side: Both of the groups are $\Lambda$ for $i \in[0, r]$, and the maps are multiplication by $n$. The generating class $h \in A_{r} \mathcal{O}(n)$ is the first Chern class of the pullback of the canonical line bundle from $\mathbf{P}^{r}$. Since the representation of $\mathbf{G}_{m}$ restricts to the canonical representation of $\mu_{n}, h$ restricted to $B$ is $l$. This proves (i).

For $X$ arbitrary, there are Künneth formulas $A_{*}\left(\mathbf{P}^{r} \times X\right)=A_{*} \mathbf{P}^{r} \otimes A_{*} X$ and $A_{*}(\mathcal{O}(n) \times X)=A_{*} \mathcal{O}(n) \otimes A_{*} X$. (ii) follows from (i) and the application of these formulas.

7.2. We now make two assumptions that will be in force for the remainder of the paper. Pick a prime $p$ not equal to the characteristic of $k$, and set $\Lambda=\mathbf{F}_{p}$. Chow groups written without explicit coefficients will thus be taken modulo $p$. Now, if $k^{\times}$contains the group $\mu_{p}(\bar{k})$, then $C(p) \cong \mu_{p}$. But of course the two groups are not naturally isomorphic. The isomorphisms themselves are in correspondence with the primitive $p$-th roots of unity. Therefore, let us assume that such a primitive root $\zeta$ has been chosen in $\bar{k}$. We can then write $A^{*} B C(p)_{\bar{k}}=\mathbf{F}_{p}[l]$.

Remark 7.3. Note that even without choosing $\zeta$ there is a natural correspondence between one-dimensional representations of $C(p)$ and $\mu_{p}$. The first Chern class then gives a correspondence between $\mu_{p}$ and $A^{1} B C(p)$. In other words, over a field containing the $p$-th roots of unity, $A^{1} B C(p)=\mathbf{Z} / p(1)$. This implies that $A^{k} B C(p)=\mathbf{Z} / p(k)$. 
Proposition 7.4. Let $r=\left[k\left(\mu_{p}\right): k\right]$ be the index of the extension obtained by adjoining the $p$-th roots of unity to $k$. Then $A^{*} B C(p) \cong \mathbf{F}_{p}[\epsilon]$ where $\epsilon$ is the top Chern class of an $r$-dimensional $C(p)$ representation.

Proof. Let $M=k\left(\mu_{p}\right)$ and set $G=\operatorname{Gal}(M / k)$. The action of $G$ on $\mu_{p}$ gives an identification of $G$ with a subgroup of $(\mathbf{Z} / p)^{\times}$.

The representation $R$ splits over $M$ into a direct sum $\bigoplus_{i=1}^{p-1} L^{\otimes i}$ - here $L$ is the one-dimensional representation associated to $\zeta$. Under the natural identification of $A^{1} B C(p)_{M}$ with $\mu_{p}, c_{1}\left(L^{\otimes i}\right)=\zeta^{i}$. Let $S$ be a coset of $(\mathbf{Z} / p)^{\times}$modulo $G$. The $r$-dimensional representation $V=\bigotimes_{i \in S} L^{\otimes i}$ is defined over $k$ since it is fixed by $G$.

All of the Chern classes of $V_{S}$ vanish except for the top class, which is nontrivial and thus a generator of $A^{r} B C(p)$. We claim that $\epsilon=c_{\text {top }}(V)$ generates $A^{*} B C(p)$.

Let $\rho: A^{*} B C(p)_{k} \rightarrow A^{*} B C(p)_{M}$ be the pullback, and let $N: A^{*} B C(p)_{M} \rightarrow$ $A^{*} B C(p)_{k}$ be the norm map. Then $N \circ \rho$ is simply multiplication by $r$. Since $r$ is relatively prime to $p, \rho$ is a split injection. We use this injection to consider $A^{*} B C(p)$ as a subgroup of $A^{*} B C(p)_{M}$. Clearly $\epsilon$ maps to some nonzero multiple of $l^{r}$. Thus to prove the proposition it is enough to show that $A^{*} B C(p) \subset F_{p}\left[l^{r}\right]$.

Now, under the identification of $G$ with an order $r$ subgroup of $(\mathbf{Z} / p)^{\times}$, the action of an element $\alpha$ on $l^{i} \in A^{i} B C(p)_{M}$ is simply multiplication by $\alpha^{i}$. It follows that $\left(A^{*} B C(p)_{M}\right)^{G}=\mathbf{F}_{p}\left[l^{r}\right]$. Thus $A^{*} B C(p) \subset F_{p}\left[l^{r}\right]$.

Remark 7.5. If $X$ is a space on which $C(p)$ acts trivially, $A_{*}^{C(p)} X$ is a direct summand of $A_{*}^{C(p)} X_{M}$. This follows from the same norm argument used in the proof of the proposition.

Let $V$ be a vector bundle on $X$ and assume that both $V$ and $X$ have trivial $C(p)$ actions. Let $\lambda_{1}, \cdots, \lambda_{d}$ be the Chern roots of $V$, and let $c_{*}(V)=\prod\left(1+\lambda_{i}\right)$. (We will employ the standard abuses of the splitting principle here and in the sequel.) Set $w(V)=\prod_{i=1}^{d}\left(1+\lambda_{i}^{p-1}\right)$. This corresponds to the class $w$ of the introduction. In order to keep track of the degrees, we also consider the polynomial $w(V, t)=$ $\prod_{i=1}^{d}\left(1+t \lambda_{i}^{p-1}\right)$.

It is convenient to localize the Chow ring $A^{*} B C(p)$ by inverting the element $\epsilon$. Note that, since $\epsilon$ is a nonzero divisor, $A^{*} B C(p)$ injects into $\left(A^{*} B \mu_{p}\right)_{\epsilon}$. Under the assumption that $X$ has trivial $C(p)$-action, $\epsilon$ is also a nonzero divisor on $A_{*}^{C(p)} X$. Let $\eta$ denote the top Chern class $c_{\text {top }}(R)$. By Wilson's theorem, it follows that $\eta=-l^{p-1}$. Note that localizing by $\eta$ always has the same effect as localizing by $\epsilon$. Given that it is generally harmless, we will sometimes write a formula in the localized groups without mentioning explicitly which group we are working in.

Proposition 7.6. With $V$ and $X$ as above,

$$
c_{\mathrm{top}}(R \otimes V)=\eta^{d} w(V, 1 / \eta)
$$


Proof. The Chern roots of $R$ are simply $l, 2 l, \ldots,(p-1) l$. Therefore,

$$
\begin{aligned}
c_{\mathrm{top}}(R \otimes V) & =\prod_{i=1}^{d} \prod_{a=1}^{p-1}\left(\lambda_{i}+a l\right) \\
& =\prod_{i=1}^{d}\left(\lambda_{i}^{p-1}-l^{p-1}\right) \\
& =\prod_{i=1}^{d}\left(\lambda_{i}^{p-1}+c_{\mathrm{top}}(R)\right) \\
& =c_{\mathrm{top}}(R)^{d} w\left(V, c_{\mathrm{top}}(R)^{-1}\right) .
\end{aligned}
$$

In the sequel, let $c_{\text {top }}(R \otimes V)(t)$ be the (unique) polynomial in $t$ with coefficients in $A^{*} X$ such that $c_{\text {top }}(R \otimes V)(\eta)=c_{\text {top }}(R \otimes V)$.

\section{Definition and Basic Properties}

In what follows, we will write $C$ for $C(p)$ considered as a subgroup of $S=S(p)$ in the obvious way. We will also write $D^{W}$ for $D_{C}^{W}$. Note that, if $C \leq G \leq S(p)$, the restriction map $A_{*}^{G} X \rightarrow A_{*}^{C} X$ is a split injection. (The splitting is given up to a factor of $[G: C]$ by the transfer.) Thus to compute $D_{G}$ for all such $G$ it suffices to compute $D^{W}$.

We first show that $D^{W}$ is a group homomorphism, beginning with the Chow group analogue of a lemma of Steenrod. Here and for the remainder of the paper, $X$ will be a space with trivial $C$-action and $S(p)$ will act on $X^{p}$ via permutations.

Lemma 8.1. Let $T: A_{*}^{C} X^{p} \rightarrow A_{*} X^{p}$ be the transfer. Then $\Delta_{C}^{!} T=0$.

Proof. By (50), there is a commutative diagram

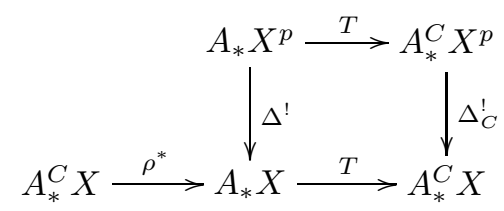

It follows from the computation of $A_{*}^{C} X$ that $\rho^{*}$ is surjective. However, $T \rho^{*}=0$ (because it is multiplication by $p$ ). Therefore, the $T$ on the bottom row is 0 . The result then follows from the commutativity of the diagram.

Theorem 8.2. $D^{W}$ is a group homomorphism.

Proof. Let $Z_{0}$ and $Z_{1}$ be two cycles. Then

$$
\left(Z_{0}+Z_{1}\right)^{\times p}=Z_{0}^{\times p}+Z_{1}^{\times p}+\Gamma
$$

where $\Gamma$ lies in the image of $T$ (by Proposition 4.7). Therefore,

$$
\Delta_{C}^{!}\left(\left(Z_{0}+Z_{1}\right)^{\times p}\right)=\Delta_{C}^{!}\left(Z_{0}^{\times p}\right)+\Delta_{C}^{!}\left(Z_{1}^{\times p}\right)
$$

by the lemma. The theorem then follows from the definition of $D^{W}$. 
Now we can view $D^{W}(\alpha)$ as a polynomial $\sum b_{i} l^{i}$ in the variable $l$ with coefficients in $A_{*} X$. We can do this even if $k$ does not contain all of the $p$-th roots of unity, because $A_{*}^{C} X \subset A_{*}^{C} X_{\bar{k}}$. Following Steenrod, we have the following.

Theorem 8.3. All terms of $b_{i} l^{i}$ of $D^{W}(\alpha)$ with $i$ not divisible by $p-1$ are 0 .

Proof. Let $G$ be the normalizer of $C$ in $S$. Then $G \cong C(p-1) \ltimes C$ with $C(p-1)$ acting through the identification $C(p-1) \cong(\mathbf{Z} / p)^{*}$. Thus $C(p-1)$ acts on $A^{*} B C$. Under the above identification, with $k \in(\mathbf{Z} / p)^{*}, k_{*} l=k l$. Thus $k_{*} l^{i}=k^{i} l^{i}$. This gives the action of $C(p-1)$ on $A^{i} B C$. Note that the action is only trivial if $i$ is a multiple of $p-1$.

Now recall that $d_{C}^{W}=\Delta_{C}^{!} \alpha^{\times p}$ is the restriction of $\Delta_{G}^{!} \alpha^{\times p}$. Therefore, $C(p-1)$ must act trivially on $D_{C}^{W}(\alpha)$ (by Proposition 3.4). Hence, all terms $b_{i} l^{i}$ with $i$ not a multiple of $p-1$ must be 0 .

8.4. Now $D^{W}(\alpha)$ can be viewed as a polynomial in the top Chern class $\eta=c_{\text {top }}(R)$. To better keep track of degrees, we let $D^{W}(\alpha, t)$ be the polynomial in $A_{*} X \otimes \mathbf{F}_{p}[t]$ such that $D^{W}(\alpha, \eta)=D^{W}(\alpha)$.

Definition 8.5. The total Steenrod operation series of the pair $(W, X)$ is the Laurent series (with finitely many terms)

$$
S_{\bullet}^{W} \alpha(t)=t^{d-k} D^{W} \alpha(1 / t)
$$

with $d=\operatorname{dim} W$ and $\alpha \in A_{k} X$. We simply write $S_{\bullet}^{W} \alpha$ for $S_{\bullet}^{W} \alpha(1)$. This is the total Steenrod operation. We define individual operations $S_{i}^{W}$ by setting $S_{\bullet}^{W} \alpha(t)=$ $\sum S_{i}^{W}(\alpha) t^{i}$. Note that $S_{i}^{W}$ lowers the degree of $\alpha$ by $(p-1) i$.

Remark 8.6. To agree with the topological notation, we define $P_{i}^{W} \alpha=S_{i}^{W} \alpha$ for $p \neq 2$. For $p=2$, we define $\mathrm{Sq}_{2 i}^{W} \alpha=S_{i}^{W}$. The odd-order operations obviously do not exist in the context of Chow groups (although they definitely do exist in the context of motivic cohomology) because the cycle class map sends Chow groups to even-dimensional cohomology.

Remark 8.7. Since $D^{W} \alpha$ is a polynomial in $t, S_{i}^{W} \alpha=0$ for $i>d-k$.

Remark 8.8. We can also formulate the definition for $\alpha \in A^{k}[W, X]$ by following through the re-indexing. We write $S_{W}^{\bullet} \alpha(t)=t^{k} D^{W} \alpha(1 / t)$ and set $S_{W}^{\bullet} \alpha(t)=$ $\sum S_{W}^{i} t^{i}$. Then, of course, $S_{W}^{i} \alpha \in A^{k+(p-1) i}[W, X]$.

From Proposition 6.5, we have

Proposition 8.9. If $j: U \rightarrow X$ is an inclusion of a Zariski open set, then $j^{*}\left(S_{\bullet}^{W} \alpha\right)=S_{\bullet}^{W}\left(j^{*} \alpha\right)$.

Proposition 8.10. If $W_{1}$ and $W_{2}$ are two smooth spaces containing $X$, then

$$
S_{\bullet}^{W_{1}} \alpha \cap w\left(T W_{2}\right)=S_{\bullet}^{W_{2}} \alpha \cap w\left(T W_{1}\right) .
$$

Proof. The proposition follows by applying successively Proposition 6.3, Proposition [7.6] and the definitions.

Definition 8.11. If $X$ is smooth, let $S^{\bullet} \alpha$ denote $S_{\bullet}^{X} \alpha$. It is natural to consider this as an operation on $A^{*} X$, so that $S^{i}$ raises degrees by $(p-1) i$. 
Remark 8.12. Suppose $f: X \rightarrow Y$ is a morphism of smooth varieties. Then from Proposition 5.3 and the functoriality of the Gysin it follows that $f^{!} D^{Y}(\alpha)=$ $D^{X}\left(f^{!} \alpha\right)$ for $\alpha \in A_{*} Y$. Therefore,

$$
f^{!} S^{\bullet} \alpha=S^{\bullet} f^{!} \alpha
$$

Definition 8.13. For any $X$ embedded in any smooth $W$, define

$$
S_{\bullet} \alpha(t)=S_{\bullet}^{W} \alpha(t) \cap w(T W, t)^{-1} .
$$

It follows from Proposition 8.10 that $S_{\bullet} \alpha$ is independent of $W$.

\section{Functorialities}

Let $i: X \rightarrow Y$ be a closed embedding with $Y$ embedded in a smooth variety $W$. Then $D_{G}^{W}\left(i_{*}(\alpha)\right)=i_{*}\left(D_{G}^{W}(\alpha)\right)$. This follows from [4, Theorem 6.2. Thus $S_{\bullet}^{W}\left(i_{*} \alpha\right)=i_{*}\left(S_{\bullet}^{W} \alpha\right)$. From the projection formula, it follows easily that $S_{\bullet}\left(i_{*} \alpha\right)=$ $i_{*}\left(S_{\bullet} \alpha\right)$.

Lemma 9.1. If $X \rightarrow U$ and $Y \rightarrow V$ are embeddings of $X$ and $Y$ into smooth varieties, then

$$
D^{U \times V}(\alpha \times \beta)=D^{U} \alpha \times D^{V} \beta .
$$

Proof. This follows directly from Example 6.5.2 of [4].

Theorem 9.2 (Cartan Formula). If $\alpha \in A_{*} X$ and $\beta \in A_{*} Y$, then

$$
\begin{aligned}
S_{\bullet}^{U \times V}(\alpha \times \beta) & =S_{\bullet}^{U} \alpha \times S_{\bullet}^{V} \beta, \\
S_{\bullet}(\alpha \times \beta) & =S_{\bullet} \alpha \times S_{\bullet} \beta .
\end{aligned}
$$

Proof. The first equation follows directly from the lemma. The second is a consequence of the multiplicative property of $w: w(T(U \times V))=w(T U) \cup w(T V)$.

Applying Remark 8.12 to the embedding $X \rightarrow X \times X$, we have the following theorem as a corollary.

Theorem 9.3 (Cartan Formula). For $X$ smooth, $D^{X}(\alpha \cup \beta)=D^{X}(\alpha) \cup D^{X}(\beta)$, and $S^{\bullet}(\alpha \cup \beta)=S^{\bullet} \alpha \cup S^{\bullet} \beta$.

Proposition 9.4. Let $X$ be a smooth space contained in a smooth space $W$ with normal bundle $N=\left(T W_{\mid X}\right) / T X$. Let $\alpha \in A^{k} X$.

(i) $D_{G}^{X}[X]=[X]$ for any group $G \leq S(n)$.

(ii) $S_{\bullet}^{\bullet}[X]=[X]$.

(iii) $S_{\bullet}^{W}[X]=[X] \cap w(N)$ and $S_{\bullet}[X]=[X] \cap w(T X)^{-1}$.

(iv) $S^{i} \alpha=\left\{\begin{array}{rr}\alpha^{p}, & i=k, \\ 0, & i>k .\end{array}\right.$

Proof. (i) is a direct consequence of the following fact: If $j: A \rightarrow B$ is a regular embedding of smooth varieties, then $j^{!}[B]=A$. Also, (i) $\Rightarrow$ (ii) by the definition of $S^{\bullet}$, and (ii) $\Rightarrow$ (iii) by Proposition 8.10 .

The second line of (iv) follows from Remark 8.7 For the first line, note that $S^{k} \alpha$ is simply the constant coefficient in $D^{X}(\alpha, t)$, that is, $S^{k} \alpha=D_{C}^{X}(\alpha, 0)$ where $C$ is the cyclic group with $p$ elements. From the functoriality of $D_{G}^{X}$ as a functor of the group $G$ under the restriction map, it follows that $D_{C}^{X}(\alpha, 0)=D_{\{1\}}^{X}(\alpha)$. But $D_{\{1\}}^{X}(\alpha)=\alpha^{p}$. 
Remark 9.5. A consequence of (iv) of the above is that, if $\alpha \in A^{1} X$ and $X$ is smooth, then $S^{\bullet}(\alpha)=\alpha+\alpha^{p}$. This implies that $D^{X}(\alpha)=\alpha\left(\eta+\alpha^{p-1}\right)$.

Corollary 9.6. For any $X$ embedded in a smooth space $W$ of dimension $d$ and $\alpha \in A_{k} X$, we have $S_{i}^{W} \alpha=0$ for $i \notin[0, d-k]$, and $S_{0}^{W}$ is the identity.

Proof. That $S_{i}^{W} \alpha$ vanishes for $i>d-k$ is Remark 8.7. To prove the rest of the corollary, first note that by linearity it suffices to consider the case $\alpha=[V]$ for $V$ an irreducible subspace. Let $V_{\text {sing }}$ be the singular locus of $V$ and let $W_{\mathrm{sm}}=W-V_{\text {sing }}$ (resp. $V_{\mathrm{sm}}=V-V_{\text {sing }}, X_{\mathrm{sm}}=X-V_{\text {sing }}$ ).

The smoothness of $V_{\mathrm{sm}}$ and Proposition 9.4 together imply that the corollary holds for $S_{i}^{W_{\mathrm{sm}}}\left[V_{\mathrm{sm}}\right]$ considered as an element of $A_{*} V_{\mathrm{sm}}$. Then the covariant functoriality of $S_{\bullet}^{W_{\mathrm{sm}}}$ shows that the corollary holds for $S_{i}^{W_{\mathrm{sm}}}\left[V_{\mathrm{sm}}\right]$ considered to be in $A_{*} X_{\mathrm{sm}}$.

Let $j: X_{\mathrm{sm}} \rightarrow X$ be the inclusion. Then by Proposition 6.5 $S_{i}^{W_{\mathrm{sm}}}\left[V_{\mathrm{sm}}\right]=$ $j^{*}\left(S_{i}^{W}[V]\right)$. The result then follows from the fact that, for $r \geq k, A_{r} X=A_{r} X_{\mathrm{sm}}$.

Lemma 9.7. Let $V$ be a vector bundle on $X$ of rank $r$ and let $\alpha \in A_{*} X$. Then $S_{\bullet}^{W}\left(\alpha \cap c_{\mathrm{top}}(V)\right)=S_{\bullet}^{W}(\alpha) \cap c_{\mathrm{top}}(V) w(V)$.

Proof. Let $P=P_{C}^{n}$. By Corollary [5.4, $P\left(\alpha \cap c_{\text {top }}(V)\right)=P(\alpha) \cap c_{\text {top }}(P(V))$. It follows from the compatibility of the refined Gysin with pullback of vector bundles (4], Proposition 6.3) that $D^{W}\left(\alpha \cap c_{\text {top }}(V)\right)=D^{W}(\alpha) \cap c_{\text {top }}(V \otimes \tilde{R})$. But, now, $c_{\text {top }}(V \otimes \tilde{R})=c_{\text {top }}(V) c_{\text {top }}(V \otimes R)$, and this is just $c_{\text {top }}(V) \eta^{r} w(V, 1 / \eta)$. By the definition of $S_{\bullet}^{W}$, we thus have $S_{\bullet}^{W}\left(\alpha \cap c_{\mathrm{top}}(V)\right)(t)=S_{\bullet}^{W}(\alpha) \cap c_{\mathrm{top}}(V) w(V, t)$, as desired.

Remark 9.8. For a line bundle $L$ with $c=c_{1}(L)$, this reduces to the statement that $S_{\bullet}^{W}(\alpha \cap c)=S_{\bullet}^{W}(\alpha) \cap\left(c+c^{p}\right)$.

In the next lemma and what follows, we use the usual convention that

$$
\left(\begin{array}{l}
n \\
k
\end{array}\right)=\frac{n(n-1) \cdots(n-k+1)}{k !}
$$

for $n \in \mathbf{Z}$ and $k \geq 0$.

Lemma 9.9. $\left(\begin{array}{c}-1-(p-1) k \\ k\end{array}\right)=0$ modulo $p$ unless $k=0$.

Proof. $\left(\begin{array}{c}-1-(p-1) k \\ k\end{array}\right)=(-1)^{k}\left(\begin{array}{c}p k \\ k\end{array}\right)$.

For a cycle $\alpha \in A_{*} X$, we write $[\alpha]_{k}$ for the component in degree $k$.

Lemma 9.10. $\left(S_{\bullet}\left[\mathbf{P}^{n}\right]\right)_{0}=0$ for $n \neq 0$.

Proof. We have $S_{\bullet}\left[\mathbf{P}^{n}\right](t)=\left[\mathbf{P}^{n}\right] \cap w\left(T \mathbf{P}^{n}, t\right)^{-1}$, and $w\left(T \mathbf{P}^{n}, t\right)=\left(1+t h^{p-1}\right)^{n+1}$. Thus, clearly, $\left(S_{\bullet}\left[\mathbf{P}^{n}\right]\right)_{0}=0$ unless $n=(p-1) k$ for some integer $k$, and, in this case, $\left(S_{\bullet}\left[\mathbf{P}^{n}\right]\right)_{0}=\left(\begin{array}{c}-n-1 \\ k\end{array}\right)$. The result then follows from Lemma 9.9

Proposition 9.11. Suppose $f: X \rightarrow Y$ factors as a closed embedding $g: X \rightarrow$ $\mathbf{P}^{n} \times Y$ followed by the projection $p_{2}: \mathbf{P}^{n} \times Y \rightarrow Y$. Then $S_{\bullet}\left(f_{*} \alpha\right)=f_{*}\left(S_{\bullet} \alpha\right)$. 
Proof. Since we know that $S_{\bullet}$ is covariantly functorial for closed embeddings, we need only show that $S_{\bullet}$ commutes with $p_{2 *}$. Let $\alpha \in A_{k}\left(Y \times \mathbf{P}^{n}\right)$. We can write $\alpha=\sum_{i+j=k} \beta_{i} \otimes\left[\mathbf{P}^{j}\right]$. Then $p_{2 *}(\alpha)=\beta_{k}$. Using the lemma and the Cartan formula, it is easy to see that $p_{2 *}\left(S_{\bullet} \alpha\right)=S_{\bullet} \beta_{k}$.

Corollary 9.12. When $k=\mathbf{C}$, the definition of $S_{\bullet}$ agrees with the topological definition given in the introduction.

Proof. Let $\pi: M \rightarrow X$ be a resolution of singularities, in particular, a projective map with $M$ smooth. Then the functoriality of $S$. for projective morphisms and the computation of Proposition 9.4 show that $S_{\bullet}[X]=\pi_{*}\left([M] \cap w(T M)^{-1}\right)$.

Of course, the proof also shows that $\pi_{*}\left([M] \cap w(T M)^{-1}\right)$ is independent of $M$.

\section{Chow Envelopes}

We now have $S$. defined for any scheme $X$ that can be embedded in a smooth scheme. In particular, it is defined for any quasi-projective scheme. Moreover, by Proposition 9.11, $S_{\bullet}$ is covariant for projective morphisms. To extend the definition

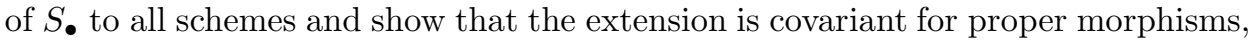
we use a Chow envelope argument essentially identical to the one used by Fulton [4] to extend the Grothendiek-Riemann-Roch theorem from quasi-projective varieties to arbitrary schemes.

We begin by reviewing the theory of Chow envelopes, referring the reader to section 18.3 of [4] for details. If $X$ is a scheme, an envelope of $X$ is a proper morphism $p: X^{\prime} \rightarrow X$ such that, for any irreducible subscheme $V$ of $X$, there is an irreducible subscheme $V^{\prime}$ of $X^{\prime}$ such that $p$ maps $V^{\prime}$ birationally onto $V$. It follows that $p_{*}: A_{*} X^{\prime} \rightarrow A_{*} X$ is surjective. An envelope $X^{\prime}$ is a Chow envelope if $X^{\prime}$ is quasi-projective. By Lemma 18.3 of [4], for any scheme $X$ there are a Chow envelope $p: X^{\prime} \rightarrow X$ and a closed subscheme $Y$ such that $X-Y$ is dense and $p$ maps $X^{\prime}-p^{-1} Y$ isomorphically onto $X-Y$.

Suppose that $p: X^{\prime} \rightarrow X$ is a Chow envelope and that $\alpha \in A_{k} X$ is a cycle class. Since we can find an $\alpha^{\prime} \in A_{k} X^{\prime}$ such that $p_{*} \alpha^{\prime}=\alpha$, the natural inclination is to simply define

$$
S_{\bullet} \alpha=p_{*} S_{\bullet} \alpha^{\prime} .
$$

However, we must first prove that this definition is independent of the choice of $\alpha^{\prime}$ and the choice of $X^{\prime}$. Following Fulton, we will say that a map $S: A_{*} X \rightarrow A_{*} X$ is compatible with $p$ if

$$
S p_{*} \alpha^{\prime}=p_{*} S_{\bullet} \alpha^{\prime}
$$

for any $\alpha^{\prime} \in A_{*} X^{\prime}$. Since $p: X^{\prime} \rightarrow X$ is an envelope, there can be at most one map $S$ compatible with a given $p$.

Lemma 10.1. If $S: A_{*} X \rightarrow A_{*} X$ is compatible with a Chow envelope $p$, then, for any proper morphism $f: Y \rightarrow X$ with $Y$ quasi-projective, the diagram

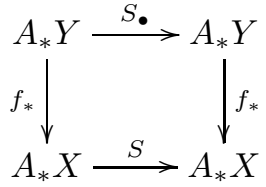

commutes. In particular, $S$ is compatible with any other Chow envelope. 
Proof. By Lemma 18.3 (4) of [4, we can find a Chow envelope $q: Y^{\prime} \rightarrow Y$ and a proper morphism $f^{\prime}: Y^{\prime} \rightarrow X^{\prime}$ such that $p f^{\prime}=f q$. Let $\alpha \in A_{k} Y$. Since $Y^{\prime}$ is an envelope, we have $\alpha=q_{*} \alpha^{\prime}$ for some $\alpha^{\prime} \in A_{k} Y^{\prime}$. Then $f_{*} S_{\bullet} \alpha=f_{*} S_{\bullet} q_{*} \alpha^{\prime}=$ $f_{*} q_{*} S_{\bullet} \alpha^{\prime}$, since $q$ is projective. The fact that $S$ is compatible with $p$ then implies that $f_{*} q_{*} S_{\bullet} \alpha=p_{*} f_{*}^{\prime} S \bullet \alpha^{\prime}=S p_{*} f_{*}^{\prime} \alpha^{\prime}=S f_{*} \alpha$.

Proposition 10.2. For any scheme $X$, there is an $S: A_{*} X \rightarrow A_{*} X$ compatible with all Chow envelopes.

Proof. We prove the proposition by Noetherian induction on $X$. When the dimension of $X$ is 0 , the proposition is trivial. Therefore, assume the proposition holds for all schemes of dimension less than that of $X$. By Lemma 10.1, it suffices to find a specific envelope $p$ and construct an $S$ compatible with $p$. We can thus assume that $p: X^{\prime} \rightarrow X$ is a Chow envelope equipped with a closed subscheme $Y$ such that $X-Y$ is dense and $p$ maps $X^{\prime}-p^{-1} Y$ isomorphically onto $X-Y$.

We then have a fiber square

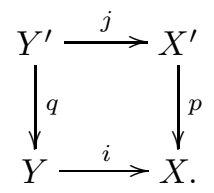

Note that $q: Y^{\prime} \rightarrow Y$ is also a Chow envelope by Lemma 18.3 (2) of [4].

By 4], Example 1.8.1, the sequence

$$
A_{k} Y^{\prime} \stackrel{a}{\rightarrow} A_{k} Y \oplus A_{k} X^{\prime} \stackrel{b}{\rightarrow} A_{k} X \rightarrow 0
$$

is exact, where $a(\gamma)=\left(q_{*} \gamma,-j_{*} \gamma\right)$ and $b(\alpha, \beta)=i_{*} \alpha+p_{*} \beta$.

From the induction hypothesis, there is a map $S: A_{*} Y \rightarrow A_{*} Y$ compatible with $q$. We can now define $S\left(i_{*} \alpha+p_{*} \beta\right)=i_{*} S(\alpha)+p_{*} S_{\bullet}(\beta)$. This is well-defined, because, for $\gamma \in A_{k} Y^{\prime}, i_{*} S\left(q_{*} \gamma\right)-p_{*} S_{\bullet}\left(j_{*} \gamma\right)=i_{*} q_{*} S_{\bullet} \gamma-p_{*} j_{*} S_{\bullet} \gamma=0$.

We can thus define $S_{\bullet}: A_{*} X \rightarrow A_{*} X$ to be given by the (necessarily unique) $S$ compatible with all Chow envelopes. By Lemma 10.1, we know that $S_{\bullet}$ commutes with proper pushforward from projective schemes. If $f: Y \rightarrow X$ is an arbitrary proper morphism, we can (as in the proof of the proposition) find Chow envelopes $q: Y^{\prime} \rightarrow Y$ and $p: X^{\prime} \rightarrow X$ and a proper map $f^{\prime}: Y^{\prime} \rightarrow X^{\prime}$ such that $p f^{\prime}=f q$. Then, if $\alpha \in A_{k} Y$ is a class, we can find an $\alpha^{\prime} \in A_{k} Y^{\prime}$ such that $q_{*} \alpha^{\prime}=\alpha$. It follows then that $f_{*} S_{\bullet} \alpha=f_{*} S_{\bullet} q_{*} \alpha^{\prime}=p_{*} f_{*}^{\prime} S_{\bullet} \alpha^{\prime}=S_{\bullet} p_{*} f_{*}^{\prime} \alpha^{\prime}=S_{\bullet} f_{*} \alpha$. We thus have the following fact.

Proposition 10.3. If $f: Y \rightarrow X$ is a proper morphism, then $f_{*} S_{\bullet}=S_{\bullet} f_{*}$.

Note that the above proposition together with Proposition 9.4(iii) characterizes the action of $S_{\bullet}$ on any variety $X$ that admits an envelope $f: Y \rightarrow X$ with $Y$ smooth. In particular, in characteristic 0 where resolution of singularities is available, $S_{\bullet}$ is characterized by commutativity with proper morphisms and the fact that $S_{\bullet}[Y]=[Y] \cap w(T Y)^{-1}$ for smooth $Y$.

Proposition 10.4. Let $X$ and $Y$ be schemes.

(i) For $\alpha, \beta \in A_{k} X, S_{\bullet}(\alpha+\beta)=S_{\bullet} \alpha+S_{\bullet} \beta$.

(ii) For $\alpha \in A_{k} X$ and $\beta \in A_{j} Y, S_{\bullet}(\alpha \times \beta)=S_{\bullet} \alpha \times S_{\bullet} \beta$. 
Proof. To verify (i), choose a Chow envelope $p: X^{\prime} \rightarrow X$ and let $\alpha^{\prime}, \beta^{\prime} \in A_{k} X^{\prime}$ be classes such that $p_{*} \alpha^{\prime}=\alpha$ and $p_{*} \beta^{\prime}=\beta$. Then

$$
S_{\bullet}(\alpha+\beta)=p_{*} S_{\bullet}\left(\alpha^{\prime}+\beta^{\prime}\right)=p_{*} S_{\bullet} \alpha^{\prime}+p_{*} S_{\bullet} \beta^{\prime}=S_{\bullet} \alpha+S_{\bullet} \beta .
$$

For (ii), let $p: X^{\prime} \rightarrow X$ and $q: Y^{\prime} \rightarrow Y$ be two Chow envelopes and let $\alpha^{\prime} \in A_{k} X^{\prime}$ and $\beta^{\prime} \in A_{j} Y^{\prime}$ be chosen such that $p_{*} \alpha^{\prime}=\alpha$ and $q_{*} \beta^{\prime}=\beta$. Then $p \times q: X^{\prime} \times Y^{\prime} \rightarrow X \times Y$ is a proper morphism and $(p \times q)_{*} \alpha^{\prime} \times \beta^{\prime}=\alpha \times \beta$. Thus, by Proposition 10.3 and Theorem 9.2 ,

$$
\begin{aligned}
S_{\bullet}(\alpha \times \beta) & =S_{\bullet}\left(p_{*} \alpha^{\prime} \times q_{*} \beta^{\prime}\right)=(p \times q)_{*} S_{\bullet}\left(\alpha^{\prime} \times \beta^{\prime}\right) \\
& =(p \times q)_{*} S_{\bullet} \alpha^{\prime} \times S_{\bullet} \beta^{\prime}=p_{*} S_{\bullet} \alpha^{\prime} \times p_{*} S_{\bullet} \beta^{\prime}=S_{\bullet} \alpha \times S_{\bullet} \beta .
\end{aligned}
$$

\section{Adem Relations}

In the topological setting, the Steenrod powers along with the Bockstein element $\beta$ generate an $\mathbf{F}_{p}$-algebra $\mathcal{A}(p)$ known as the Steenrod algebra. This highly non-commutative algebra is the free non-commutative algebra on the Steenrod operations (along with the Bockstein element) modulo the two-sided ideal generated by certain (somewhat complicated) relations known as the Adem relations. The fact that the cohomology $H^{*}\left(X, \mathbf{F}_{p}\right)$ of a topological space $X$ is a module over the Steenrod algebra $\mathcal{A}(p)$ has profound consequences for algebraic topology.

In our algebraic setting, we will not have an analogue of the Bockstein element acting on Chow theory, because $\beta$ increases topological degree by 1 . (Note, however, that, in the setting of motivic cohomology, $\beta$ exists and plays essentially the same role as the Bockstein element in algebraic topology.) Thus we should expect that $\mathcal{A}(p)$ modulo the two-sided ideal generated by $\beta$ will act on the Chow groups. To prove this we must check the Adem relations.

In this section, we verify that the Adem relations (given in equation (76)) hold both for the $S_{i}^{W}$ and for the $S_{i}$. Most of the proof is formal and some of it is tedious. In general, the proof is very similar to the one given by Steenrod in [8]. I have, therefore, sketched certain arguments, referring the reader to Steenrod for the details.

In order to unify the presentation, we use the re-indexing of Remarks 6.2 and 8.8. Therefore, we will be considering throughout the groups $A^{k}[W, X]$ and the operations $S_{W}^{\bullet}=t^{k} D^{W} \alpha(1 / t)$. We will also use the symbol $\infty$ to stand for an "infinite" $W$. In practice, this means that we will write $A^{k}[\infty, X]=A_{-k} X$ and $S_{\infty}^{\bullet}(\alpha)$ for the re-indexed $S_{\bullet}(\alpha)$. We will say that $W$ is "finite" if it is not $\infty$. When $X$ is smooth, $D$ written without a superscript will mean $D^{X}$.

For $W$ finite and $\alpha \in A^{k}[W, X]$, we have $D^{W}(\alpha)=\eta^{k} S_{W}^{\bullet} \alpha(1 / \eta)$. We generalize this formula by defining $D^{\infty}(\alpha)=\eta^{k} S_{\infty}^{\bullet} \alpha(1 / \eta)$. Note that, while $D^{W}(\alpha) \in$ $A_{C}^{*}[W, X]$ for $W$ finite, $D^{\infty}(\alpha)$ may only live in the localized module $A_{C}^{*}[W, X]_{\eta}$.

Now let $C_{1}$ and $C_{2}$ be two copies of the group $C(p)$ and suppose that $E_{i} \in \mathrm{E} C_{i}$ for $i=1,2$ over an algebraically closed field $k$. (In proving the Adem relations, we can work over an algebraically closed field without loss of generality.) Let $B_{i}=E_{i} / C_{i}$ for $i=1,2$. We suppose that a large integer $N$ has been chosen and that $A^{k} B_{i}=A^{k} B C_{i}$ for $k \leq N$. Let us write $A^{*} B C_{i}=\mathbf{F}\left[l_{i}\right]$. We will also write 
$\eta_{i}=-l_{i}^{p-1}$. Let $R_{i}^{\prime}$ be the subalgebra of $A^{*} B C_{i}$ generated by $\eta_{i}$. In general, we write $R^{\prime}$ for the subalgebra of $A^{*} B C(p)$ generated by $\eta$.

We want to compute the map $D: A^{k} B_{1} \rightarrow A^{*} B_{1} \otimes A^{*} B C_{2}$. By Remark 9.5 $D\left(l_{1}\right)=l_{1}\left(\eta_{2}+l_{1}^{p-1}\right)=l_{1}\left(\eta_{2}-\eta_{1}\right)$. Since $D$ is an algebra homomorphism, this computes $D$ completely. In particular, $D\left(\eta_{1}\right)=\eta_{1}\left(\eta_{2}-\eta_{1}\right)^{p-1}$. These two formulas hold for the varieties $B_{i}$. There is then an induced algebra map $D: A^{*} B C \rightarrow$ $A^{*} B C \otimes A^{*} B C$ given by $D(l)=l \otimes 1\left(l^{p-1} \otimes 1-1 \otimes l^{p-1}\right)$.

Now for any space $X$ embedded in a smooth (finite) space $W$ there is a sequence

$$
A^{k}[W, X] \rightarrow A^{p k}\left[W \times B_{1}, X \times B_{1}\right] \rightarrow A^{p^{2} k}\left[W \times B_{1} \times B_{2}, X \times B_{1} \times B_{2}\right]
$$

obtained by applying $D^{W}$ and then $D^{W \times B_{1}}$ in succession. By taking $N>p^{2} k$, this defines a map $D^{2}: A^{*}[W, X] \rightarrow A^{*}[W, X] \otimes \mathbf{F}\left[l_{1}\right] \otimes \mathbf{F}\left[l_{2}\right]$. Let $s$ be the automorphism of $R^{\prime} \otimes R^{\prime}$ that switches the factors, i.e., $s\left(l_{1}\right)=l_{2}, s\left(l_{2}\right)=l_{1}$.

Theorem 11.1. For $W$ finite, $D^{2}=s\left(D^{2}\right)$.

Sketch of the Proof. Let $C^{2}$ be the product of $C_{1}$ and $C_{2}$ and let sw be the automorphism of $C^{2}$ that switches the factors. Then $D^{2}$ can be thought of as a map $D^{2}: A^{*}[W, X] \rightarrow A_{C^{2}}^{*}[W, X]$. Then $\mathrm{sw}^{*}\left(D^{2}\right)=D^{2}$. This is shown in the topological context on pages 116 and 117 of [8]. The proof in the context of equivariant intersection theory uses the same reasoning and the same commutative diagrams. (All of the necessary functorial properties of equivariant intersection theory are at our disposal.) I will omit it.

It remains to show that $\mathrm{sw}^{*}=s$. But this is clear from the way sw acts on one-dimensional representations.

Let $m: R^{\prime} \otimes R^{\prime} \rightarrow R^{\prime}$ be the multiplication map. Let $R=R_{\eta}^{\prime}$. Then $R$ inherits a grading from $A^{*} B C(p)$ so that $\eta$ has degree $p-1$. Extend $D, m$, and $s$ to $R$. Then, since $D\left(\eta_{1}\right)=\eta_{1}\left(\eta_{2}-\eta_{1}\right)^{p-1}$, the extension of $D$ to $R$ takes values in the ring $(R \otimes R)_{\eta_{2}-\eta_{1}}$. (Here we write $\eta_{1}$ for $\eta \otimes 1, \eta_{2}$ for $1 \otimes \eta$.) Write $M=A^{*}[W, X]$ and $D^{M}=D^{W}$. The Cartan formula shows us that $D^{2}$ can be computed as the composition

$$
\begin{array}{rll}
M \stackrel{D^{M}}{\longrightarrow} & M \otimes R^{\prime} & \stackrel{D^{M} \otimes D}{\longrightarrow} M \otimes R^{\prime} \otimes R^{\prime} \otimes R^{\prime} \\
& \stackrel{1 \otimes s \otimes 1}{\longrightarrow} & M \otimes R^{\prime} \otimes R^{\prime} \otimes R^{\prime} \stackrel{1 \otimes 1 \otimes m}{\longrightarrow} M \otimes R^{\prime} \otimes R^{\prime}
\end{array}
$$

We can then extend this to a map $D^{2}: M \rightarrow(M \otimes R \otimes R)_{\eta_{2}-\eta_{1}}$ by extending $D$ to $R$.

11.2. Let $\iota$ be the involution on $R$ taking $\eta$ to $1 / \eta$. Let $M$ be any graded $\mathbf{F}_{p}$-module with a map $D^{M}: M \rightarrow M \otimes R$ multiplying degrees by $p$. We write $D^{M}(\alpha)(1 / \eta)$ for $(\operatorname{id} \otimes \iota) \circ D^{M}(\alpha)$. Define $S_{M}(\alpha)=\eta^{k} D^{M}(\alpha)(1 / \eta)$ and write $S_{M}(\alpha)=\sum_{i} S_{M}^{i}(\alpha) \eta^{i}$. For $\alpha \in M_{k}$, note that this implies that $D^{M}(\alpha)=\eta^{k} S_{M}(\alpha)(1 / \eta)$. Define $D^{2}$ : $M \rightarrow M \otimes(R \otimes R)_{\left(\eta_{2}-\eta_{1}\right)}$ as in (75)).

The Adem relations will follow from the following theorem.

Theorem 11.3. With $M$ as above, suppose that

(i) for any $\alpha, S_{M}^{i}(\alpha)=0$ for $i<0$,

(ii) $s\left(D^{2}\right)=D^{2}$. 
Then the $S_{M}^{i}$ satisfy the Adem relations. That is, for $0<b<p c$,

$$
S_{M}^{b} S_{M}^{c}=\sum_{i=0}^{[b / p]}(-1)^{b+i}\left(\begin{array}{c}
(p-1)(c-i)-1 \\
b-p i
\end{array}\right) S_{M}^{b+c-i} S_{M}^{i} .
$$

Proof. We will write $u$ for the generator of the first factor of $R$ and $v$ for the other, so that $R \otimes R=\mathbf{F}\left[u, v, u^{-1}, v^{-1}\right]$. In other words, we write $u$ for $\eta_{1}$ and $v$ for $\eta_{2}$. We will simply write $S$ for $S_{M}$. Note that $S^{i}$ raises degrees by $(p-1) i$.

We then compute, for $\alpha \in M_{k}$,

$$
\begin{aligned}
D^{2}(\alpha) & =D\left(u^{k} S(\alpha)(1 / u)\right) \\
& =D\left(u^{k} \sum_{i \geq 0} S^{i}(\alpha) u^{-i}\right) \\
& =\sum_{i \geq 0} D\left(S^{i}(\alpha)\right) D\left(u^{k-i}\right) \\
& =\sum_{i \geq 0} v^{k+(p-1) i} \sum_{j \geq 0} S^{j} S^{i}(\alpha) v^{-j} D\left(u^{k-i}\right) \\
& =\sum_{i, j \geq 0} S^{j} S^{i}(\alpha) v^{k+(p-1) i-j} u^{k-i}(v-u)^{(p-1)(k-i)} .
\end{aligned}
$$

Note that the sums here are finite by the assumption that $D: M \rightarrow M \otimes R$.

By assumption (ii), we have that

$$
\sum_{i, j \geq 0} S^{j} S^{i}(\alpha)(v-u)^{(p-1)(k-i)}\left[v^{k+(p-1) i-j} u^{k-i}-u^{k+(p-1) i-j} v^{k-i}\right]=0 .
$$

We reduce this by dividing through by $(v-u)^{(p-1) k} v^{k} u^{k}$, to obtain the equation

$$
\sum_{i, j \geq 0} S^{j} S^{i}(\alpha)(v-u)^{-(p-1) i}\left[v^{(p-1) i-j} u^{-i}-u^{(p-1) i-j} v^{-i}\right]=0 .
$$

We then change variables, writing the equation in terms of $a=u / v$ and $v$, to obtain

$$
\sum_{i, j \geq 0} S^{j} S^{i}(\alpha) v^{-i-j}(1-a)^{-(p-1) i}\left[a^{-i}-a^{p i-i-j}\right]=0 .
$$

Thus, with $m=b+c$,

$$
\sum_{i+j=m} S^{j} S^{i}(\alpha)(1-a)^{-(p-1) i}\left[a^{-i}-a^{p i-m}\right]=0 .
$$

Multiplying through by $a^{m}$, we have

$$
\sum_{i+j=m} S^{j} S^{i}(\alpha)(1-a)^{-(p-1) i}\left[a^{j}-a^{p i}\right]=0 .
$$

In other words,

$$
\sum_{i+j=m} S^{j} S^{i}(\alpha)(1-a)^{-(p-1) i} a^{j}=\sum_{i+j=m} S^{j} S^{i}(\alpha)(1-a)^{-(p-1) i} a^{p i} .
$$

Now, multiply both sides through by $(1-a)^{(p-1) c-1} a^{-b}$ to get

$$
\begin{aligned}
& \sum_{i+j=m} S^{j} S^{i}(\alpha)(1-a)^{(p-1)(c-i)-1} a^{j-b} \\
&=\sum_{i+j=m} S^{j} S^{i}(\alpha)(1-a)^{(p-1)(c-i)-1} a^{p i-b} .
\end{aligned}
$$


The Adem relations follow from considering the constant coefficient of each side of the equation expanded out as a formal power series in $a$.

On the left side, the coefficient is simply $S^{b} S^{c}$. This follows from Lemma 9.9 Note that on the right the coefficient will be 0 unless $p i \leq b$. Since $0<b<p c$, this implies that $i<c$. At any rate, the coefficient is

$$
\sum_{i+j=m} S^{j} S^{i}(-1)^{b-p i}\left(\begin{array}{c}
(p-1)(c-i)-1 \\
b-p i
\end{array}\right) .
$$

The theorem directly implies the Adem relations for finite $W$. For $W=\infty$, one needs to know the following fact.

Proposition 11.4. If $D^{W}$ satisfies the conditions of Theorem 11.3 for any finite $W$, then $D^{\infty}$ does also.

Proof. Hypothesis (i) of Theorem 11.3 holds automatically, since $S_{i} \alpha$ vanishes for $i<0$. (This is implied by the vanishing of $S_{i}^{W} \alpha$ for $i<0$.) The proof of (ii) is a combinatorial game with the Chern roots of $T W$. Writing them as $\lambda_{i}$, we have

$$
w(T M)=\prod_{i=1}^{d}\left(1+\lambda_{i}^{p-1}\right) .
$$

Now, $D^{W} \alpha=\eta^{k} S_{W}^{\bullet} \alpha(1 / \eta)$ for $\alpha \in A^{k}[W, X]$. If $\lambda$ is the first Chern class of a line bundle $L$, it follows from Remark 9.8 that

$$
D^{W}(\alpha \cap \lambda)=D^{W}(\alpha) \cap D(\lambda)
$$

with $D(\lambda)=\lambda\left(\eta+\lambda^{p-1}\right)$.

Now suppose that $\alpha \in A^{k}[W, X]=A_{d-k} X=A^{k-d}[\infty, X]$. From the definition of $S$ • (taking into account the gradings), it follows that

$$
\begin{aligned}
D^{\infty} \alpha & =\eta^{k-d} S_{W}^{\bullet} \alpha(1 / \eta) \cap \prod_{i=1}^{d}\left(1+\eta^{-1} \lambda_{i}^{p-1}\right)^{-1} \\
& =D^{W} \alpha \cap \prod_{i=1}^{d}\left(\eta+\lambda_{i}^{p-1}\right)^{-1} .
\end{aligned}
$$

From the fact that $D^{W}$ is a homomorphism, we then have

$$
\begin{aligned}
D^{\infty} D^{\infty} \alpha & =D^{W}\left(D^{W} \alpha \cap \prod_{i=1}^{d}\left(\eta_{1}+\lambda_{i}^{p-1}\right)^{-1}\right) \cap \prod_{i=1}^{d}\left(\eta_{2}+\lambda_{i}^{p-1}\right)^{-1} \\
& =D^{W} D^{W} \alpha \cap D\left(\prod_{i=1}^{d}\left(\eta_{1}+\lambda_{i}^{p-1}\right)^{-1}\right) \cap \prod_{i=1}^{d}\left(\eta_{2}+\lambda_{i}^{p-1}\right)^{-1} .
\end{aligned}
$$

Assuming that (ii) holds for $W$, we know that $D^{W} D^{W} \alpha$ is symmetric in $\eta_{1}, \eta_{2}$. Therefore, it will suffice to show that the expression

$$
D\left(\prod_{i=1}^{d}\left(\eta_{1}+\lambda_{i}^{p-1}\right)^{-1}\right) \cap \prod_{i=1}^{d}\left(\eta_{2}+\lambda_{i}^{p-1}\right)^{-1}
$$


is symmetric in $\eta_{1}, \eta_{2}$. Moreover, since $D^{W}$ is an algebra homomorphism, we can assume that $d=1$, so that there is only one $\lambda=\lambda_{1}$, and we can remove the inverses. That is, we only need to show that

$$
D\left(\eta_{1}+\lambda^{p-1}\right)\left(\eta_{2}+\lambda^{p-1}\right)
$$

is symmetric for $\lambda$ a first Chern class.

We compute

$$
\begin{aligned}
D\left(\eta_{1}\right. & \left.+\lambda^{p-1}\right)\left(\eta_{2}+\lambda^{p-1}\right) \\
& =\left[\eta_{1}\left(\eta_{2}-\eta_{1}\right)^{p-1}+\lambda^{p-1}\left(\lambda^{p-1}+\eta_{2}\right)^{p-1}\right]\left[\eta_{2}+\lambda^{p-1}\right] \\
& =\eta_{1}\left(\eta_{2}-\eta_{1}\right)^{p-1}\left(\eta_{2}+\lambda^{p-1}\right)+\lambda^{p-1}\left(\lambda^{p-1}+\eta_{2}\right)^{p} \\
& =\frac{\eta_{1}\left(\eta_{2}^{p}-\eta_{1}^{p}\right)\left(\eta_{2}+\lambda^{p-1}\right)+\lambda^{p-1}\left(\eta_{2}-\eta_{1}\right)\left(\lambda^{p(p-1)}+\eta_{2}^{p}\right)}{\eta_{2}-\eta_{1}} \\
& =\frac{\eta_{1} \eta_{2}\left(\eta_{2}^{p}-\eta_{1}^{p}\right)+\left(\eta_{2}^{p+1}-\eta_{1}^{p+1}\right) \lambda^{p-1}+\left(\eta_{2}-\eta_{1}\right) \lambda^{p^{2}-1}}{\eta_{2}-\eta_{1}} .
\end{aligned}
$$

Since both the numerator and the denominator of the last expression are odd functions of $\eta_{2}-\eta_{1}$, 80 is symmetric.

As a corollary, we obtain

Theorem 11.5. Let $\mathcal{A}(p)$ be the Steenrod algebra at the prime $p$, and let $\beta$ be the Bockstein element. Then $\mathcal{A}(p)$ modulo the two-sided ideal generated by $\beta$ acts on $A^{*}[W, X]$ for $W$ either a smooth algebraic space in which $X$ is embedded or $W=\infty$.

\section{ACKNOWLEDGMENTS}

Most of the work described in this paper was done at the University of Chicago as part of my 1998 thesis. I would like to thank S. Bloch, W. Fulton and B. Totaro for guidance and encouragement there. I would also like to thank A. Merkurev for catching an error in a previous version of this paper.

\section{REFERENCES}

1. M. Artin. Versal deformations and algebraic stacks. Invent. Math., 27:165-189, 1974. MR 53:2945

2. P. Deligne and D. Mumford. The irreducibility of the space of curves of given genus. Inst. Hautes Études Sci. Publ. Math., (36):75-109, 1969. MR 41:6850

3. Dan Edidin and William Graham. Equivariant intersection theory. Invent. Math., 131(3):595634, 1998. MR 99j:14003a

4. William Fulton. Intersection theory. Springer-Verlag, Berlin, second edition, 1998. MR 99d:14003

5. Henri Gillet. Intersection theory on algebraic stacks and $Q$-varieties. In Proceedings of the Luminy conference on algebraic K-theory (Luminy, 1983), volume 34, pages 193-240, 1984. MR 86b:14006

6. S. Kawai. A note on Steenrod reduced powers of algebraic cocycles. In Complex analysis and algebraic geometry, pages 375-381. Iwanami Shoten, Tokyo, 1977. MR 56:8562

7. John W. Milnor and James D. Stasheff. Characteristic classes. Princeton University Press, Princeton, NJ, 1974. Annals of Mathematics Studies, No. 76. MR 55:13428

8. N. E. Steenrod. Cohomology operations. Princeton University Press, Princeton, NJ, 1962. MR 26:3056 
9. Burt Totaro. The Chow ring of a classifying space. In Algebraic K-theory (Seattle, WA, 1997), Proc. Sympos. Pure Math., vol. 67, pages 249-281. Amer. Math. Soc., Providence, RI, 1999. MR 2001f: 14011

10. V. Voevodsky. The Milnor conjecture. K-theory preprint server.

11. Vladimir Voevodsky. Reduced power operations in motivic cohomology. arXiv:math. AG/0107109.

Department of Mathematics, University of California, los Angeles, California

E-mail address: pbrosnan@math.ucla.edu 